\title{
Benefits of extended capabilities of the driver accelerator for EURISOL
}

\author{
K.-H. Schmidt, ${ }^{1}$ A. Kelić, ${ }^{1}$ S. Lukić, ${ }^{1}$ M. V. Ricciardi, ${ }^{1}$ and M. Veselsky ${ }^{2}$ \\ ${ }^{1}$ Gesellschaft für Schwerionenforschung m.b.H., Planckstraße 1, 64291 Darmstadt, Germany \\ ${ }^{2}$ Institute of Physics, Slovak Academy of Sciences, Bratislava, Slovakia
}

(Received 11 October 2006; published 31 January 2007)

\begin{abstract}
Possibilities are studied for the optimization of EURISOL rare nuclide yields in specific regions of the nuclear chart by building the driver accelerator in a way that enables accelerating several additional beam species, to specific energies, besides the baseline $1 \mathrm{GeV}$ proton beam. Nuclide production rates with these driver beams are compared to the production rates expected with the $1 \mathrm{GeV}$ proton beam in the directproduction and the high-power-converter scenarios. Arguments are presented to show that several additional driver-beam scenarios could provide substantial benefit for the production of nuclides in specific regions of the nuclear chart. The quantitative values in this report are preliminary in the sense that they depend on assumptions on the values of some key parameters which are subject to technical development, e.g., maximum beam intensities or limits on the target heat load. The different scenarios are compared from the aspect of nuclide yields. The arguments presented here, when complemented by the corresponding technological and financial considerations, are intended to serve as a part of the basis for decisions on the design of the driver accelerator of EURISOL.
\end{abstract}

DOI: 10.1103/PhysRevSTAB.10.014701

PACS numbers: 29.25.Rm, 25.70.Mn, 25.85.-w, 24.10.-i

\section{INTRODUCTION}

Projects for future secondary-beam facilities aim at exploiting the very technological limits of the production of rare nuclide beams. Such a goal requires careful work on the optimization of the production techniques. That is an interdisciplinary task, as it involves the fields of accelerator technology, nuclear reactions, extraction techniques, target handling, and others.

EURISOL [1] is the European project for constructing a secondary-beam facility based on the ISOL approach, which should provide the highest beam intensities and which will give access to the most exotic nuclides within the technological limits. The present work is dedicated to provide arguments for optimizing the technical parameters of the driver accelerator, which is one of the most urgent problems to be solved. It has been performed within task 11, "Beam-intensity calculations," of the EURISOL design study.

Guided by the experience with the long-term operation of the ISOLDE facility [2,3], a $1 \mathrm{GeV}$ proton beam has been chosen as the baseline option for the driver accelerator. Using different target material, this option allows for producing a large number of isotopes of many elements. However, this option might not be the optimum solution for all nuclides of interest. Extended capabilities of the driver accelerator in order to provide additional beam species and more complex technical approaches might result in a benefit for producing nuclides in certain regions of the chart of the nuclides. The present report investigates this question in a systematic way. For this purpose, the benefit of extended capabilities of the driver accelerator is considered in connection with a quantitative discussion of nuclear- reaction aspects and the technical limitations of the ISOL method.

\section{TECHNICAL CONSTRAINTS FOR THE DRIVER ACCELERATOR}

Several concepts for the heavy-ion driver have been mentioned in the EURISOL report [4] resulting from the feasibility study Research and Technical Development (RTD). These concepts define the technical constraints on eventual extensions of the driver accelerator: (1) The three HI driver-beam options described in the report of the driver-accelerator group (Appendix B, Section 4.2 of the EURISOL RTD report [4]): (a) $A / q=2$ at $43 A \mathrm{MeV}$ and $A / q=3$ at $28 A \mathrm{MeV}$, (b) $A / q=2$ at $500 A \mathrm{MeV}$, (c) $A / q=3$ at $100 A \mathrm{MeV}$ and $A / q=2$ at $150 A \mathrm{MeV}$; (2) the HI driver-beam option described in the target-and-ionsource group (Appendix C, Sec. 2.2.3 of the EURISOL RTD report [4]): $A / q=6$ at $166.5 A \mathrm{MeV}$ and $A / q=3$ at $333 A \mathrm{MeV}$.

$\mathrm{A}^{3} \mathrm{He}^{2+}$ beam, accelerated to $2 \mathrm{GeV}$ in the main linac with minor cavity modifications, will also be considered.

There is an important distinction between options (1) and (2) on one side and the last option on the other side: The nuclides of interest are projectilelike residues in options (1) and (2), but targetlike residues in the last option, like in the baseline proton-beam concept.

In addition, the option of a deuteron beam of $E \leq$ $200 \mathrm{MeV}$ and a current of $\geq 0.5 \mathrm{~mA}$ for a neutronconverter option, as originally proposed by Nolen for the ANL secondary-beam facility [5-7], will be investigated.

All these options will be compared with the baseline $1 \mathrm{GeV}$ proton-driver case. This baseline option comprises 
two scenarios: (1) $200 \mu \mathrm{A}$ beam current for the directtarget option; (ii) 3-4 mA beam current for the convertertarget option.

\section{CHARACTERISTICS OF THE EURISOL BASELINE OPTION}

As an ISOL-based facility, EURISOL has to face a few technical conditions like a limited choice on the target material, element-dependent overall efficiencies, and decay losses for short-lived species. As a starting point of the present investigation, it is necessary to have a view on these characteristics. Together with the features of the intarget nuclide production by a $1-\mathrm{GeV}$ proton beam, this will allow analyzing, whether the secondary-beam production at the EURISOL facility can be enhanced in specific regions of the nuclear chart if the baseline driver-beam options ( $1 \mathrm{GeV}$ proton beam of 3-4 MW on a converter target and $200 \mathrm{~kW}$ on a direct target) are complemented by additional driver beams.

\section{A. Target material}

The two baseline proton-driver options, direct target and high-power fission target, rely on the use of a thick target, where the radioactive species are produced by the primary protons and by the secondary neutrons, respectively. Thus, it is the same material in which the radioactive species are produced and from which they have to be extracted. For this reason, at present only a limited number of elements is well suited as a component of an ISOL target, e.g., due to the required thermal stability. The following list of target materials used at ISOLDE and some other ISOL facilities has been compiled and discussed by Ulli Köster in 2001 [8]: molten metal targets - typical elements used for ISOL molten-metal targets: $\mathrm{Ge}, \mathrm{Sn}, \mathrm{La}, \mathrm{Pb}, \mathrm{Bi}, \mathrm{U}$; (ii) metal-foil targets - typical elements used for ISOL metal-foil targets are $\mathrm{Ti}, \mathrm{Nb}, \mathrm{Ta}, \mathrm{W}$; (iii) carbide targets ${ }^{1}$ - typical carbide targets are $\mathrm{C}, \mathrm{Al}_{4} \mathrm{C}_{3}, \mathrm{SiC}, \mathrm{VC}, \mathrm{LaC}_{2}, \mathrm{ThC}_{2}, \mathrm{UC}_{2}$. Other possible carbide targets are $\mathrm{ScC}_{2}, \mathrm{CaC}_{2}, \mathrm{TiC}, \mathrm{ZrC}, \mathrm{GdC}_{x}$; (iii) oxide targets - typical oxide targets are $\mathrm{MgO}, \mathrm{Al}_{2} \mathrm{O}_{3}$, $\mathrm{CaO}, \mathrm{TiO}_{2}, \mathrm{SrO}, \mathrm{ZrO}_{2}, \mathrm{BaO}, \mathrm{La}_{2} \mathrm{O}_{3}, \mathrm{CeO}_{2}, \mathrm{ThO}_{2}$; (iv) other targets - some other target material was used, at least for tests: $\mathrm{BN}, \mathrm{AlN}, \mathrm{CaB}_{6}, \mathrm{Zr}_{5} \mathrm{Si}_{3}, \mathrm{Zr}_{5} \mathrm{Ge}_{3}, \mathrm{BaB}_{6}$, $\mathrm{BaZrO}_{3}, \mathrm{CeB}_{6}, \mathrm{CeS}, \mathrm{Hf}_{3} \mathrm{Si}_{2}, \mathrm{Hf}_{5} \mathrm{Ge}_{3}, \mathrm{Hf}_{5} \mathrm{Sn}_{3}, \mathrm{Ta}_{3} \mathrm{Si}_{3}, \mathrm{Na}-$ zeolite, Ca-zeolite, and Ti-zeolite.

The restrictions are more severe than it may appear from this list because there are further restrictions on the target material for specific elements to be extracted as secondary beams, e.g., due to conditions on chemical reactivity or vapor pressure of the target material. These limitations are explicitly discussed in Ref. [8].

\footnotetext{
${ }^{1}$ Often the stochiometric carbide is diluted in excess graphite to provide more favorable release characteristics.
}

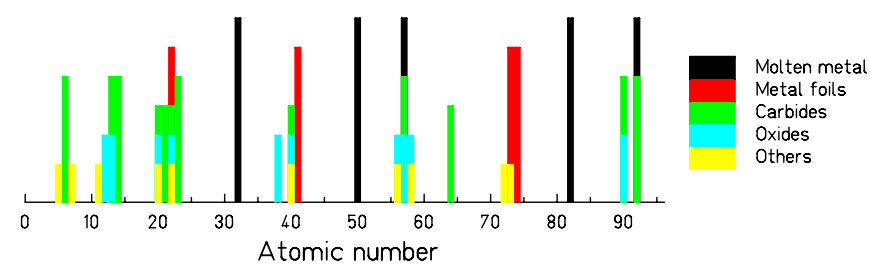

FIG. 1. (Color) Graphical presentation of the elements used for ISOL targets (data from Ref. [8]). (The columns are drawn with different heights for graphical reasons only.)

Figure 1 shows a graphical presentation, which illustrates that important gaps exist between the different elements used as ISOL targets. As a general rule, one may conclude that these gaps usually extend over about 10 elements. If specific target types are required for optimum extraction conditions of certain elements, one may even encounter gaps about 20 elements wide. This implies some restrictions on the optimization of the target material in view of nuclear-reaction aspects governing the production of specific secondary beams.

It has to be stressed that these limitations can be overcome in some cases by dedicated research and development. However, the general impression given by Fig. 1 will remain valid to a certain extent also for the operation of EURISOL.

\section{B. ISOL efficiencies, available elements}

Not all elements can be extracted from ISOL targets with high overall efficiencies. Even for sufficiently longlived isotopes, which are not affected by decay losses during the diffusion and effusion processes occurring in the target-ion-source system, losses may occur in the different stages of the extraction process. Some elements, e.g., the refractory elements, have even so low volatility that they are extracted with very low efficiencies or not observed at all. A general impression of the strong variation of the ISOL efficiency on the atomic number of the nuclear species can be obtained from Fig. 2, where the result of careful systematic measurements of the overall efficiencies of the OSIRIS target [9] is shown. New target-ion-source developments have led to higher efficiencies in the mean time. We refer to the intense study of overall efficiencies and intensities of beams for the seven NuPECC reference elements beryllium, argon, nickel, gallium, krypton, tin, and francium reported in Appendix $\mathrm{C}$ of the EURISOL RTD report [4]. However, a strong dependence on the chemical properties of the different elements as globally demonstrated in Fig. 2 remains important.

The elements, which were available as ISOLDE beams up to 1994, are shown in Fig. 3. It gives a good impression on the possibilities and limitations of the ISOL method.

It is particularly penalizing that the elements between ytterbium $(Z=39)$ and palladium $(Z=46)$ and elements around phosphorus $(Z=15)$ and chromium $(Z=24)$, for 


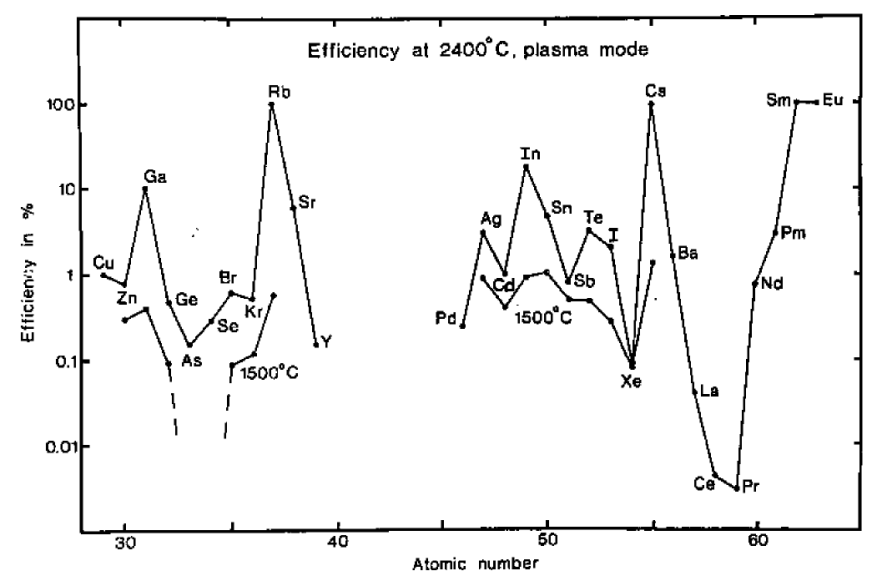

FIG. 2. Overall efficiency of the OSIRIS target and ion source as a function of atomic number of fission-product nuclei. The upper curve is measured at a target temperature of $2400{ }^{\circ} \mathrm{C}$. For comparison, the lower curve shows the efficiencies of the previously used system at $1500^{\circ} \mathrm{C}$. The figure is taken from Ref. [9].

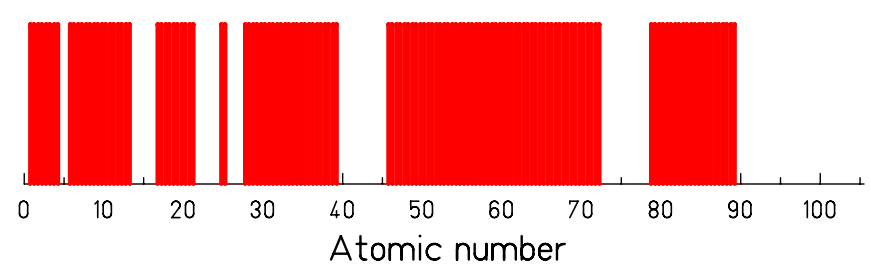

FIG. 3. (Color) Elements available as ISOLDE beams up to 1994. (Data are taken from Ref. [3].) It should be noted that the efficiencies can be rather different for the different elements.

which very neutron-rich isotopes can be produced by fission of actinides, have very low overall efficiencies.

The ISOL method relies on complex processes, with characteristics depending strongly on the element considered. Developing methods with optimized efficiency for specific elements requires intensive research, and the overall efficiencies reached are not always high, in particular, if the element has low volatility and if the half-life of the isotope of interest is short.

In order to fill some of these "gaps," a novel approach involving fragmentation and in-flight separation of a reaccelerated ISOL beam [10] is intensively investigated. However, since this scenario is not related to the driver accelerator, it is beyond the scope of the present work.

\section{Extraction times}

High primary reaction rates are reached by using rather large target volumes. Unfortunately, this leads almost inevitably to long transport times. Figure 4 illustrates the restrictions on the length of isotopic chains to be prepared as secondary beams, which are imposed by the limitations on half-lives longer than 100, 10, and $1 \mathrm{~s}$, respectively. Although the figure is incomplete, because measurements

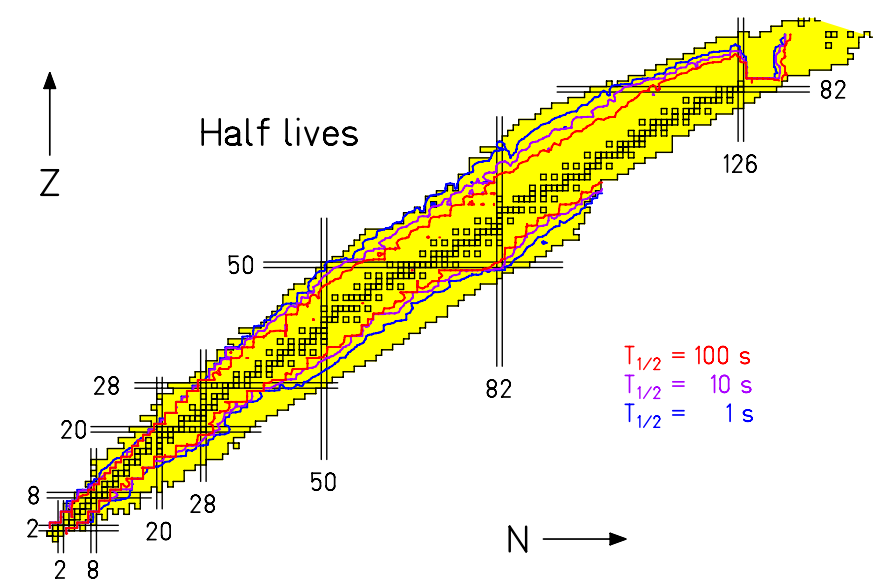

FIG. 4. (Color) Chart of the nuclides. The yellow area covers the nuclides observed up to now. Black open squares denote stable nuclides. The contour lines mark regions of equal measured nuclear life times: 1,10 , and 100 seconds. On the neutron-rich side, the contour lines are missing above $Z=65$, since the experimental data for that region do not reach short half-lives.

of shorter half-lives are scarce, the figure gives an impression on the steep decrease of the nuclear life times with increasing distance from the valley of beta stability.

Figure 5 shows a compilation of the elements for which efficiencies of the order of $1 \%$ or more are reached for half-lives above $30 \mathrm{~ms}, 1 \mathrm{~s}$, and $1 \mathrm{~min}$, respectively, from measurements at the Gesellschaft für Schwerionenforschung m.b.H, Darmstadt (GSI) mass separator. One should take into account that the targets used at the GSI mass separator were rather thin, around $1 \mathrm{mg} / \mathrm{cm}^{2}$, because they were adapted to the range of heavy ions at UNILAC energies ( $\approx 5$ to $10 \mathrm{~A} \mathrm{MeV}$ ). Extraction times from targets with larger volume as used at EURISOL can be appreciably longer.

According to a systematic analysis of ISOLDE-SC yields performed in Ref. [16], the overall efficiency $\varepsilon$ for an isotope of a given element, in function of the half-life of the isotope, can be represented by a universal analytical function:

$$
\varepsilon=\frac{\varepsilon_{s}}{1+\left(\frac{t_{1 / 2}}{\tau}\right)^{-\alpha}}
$$

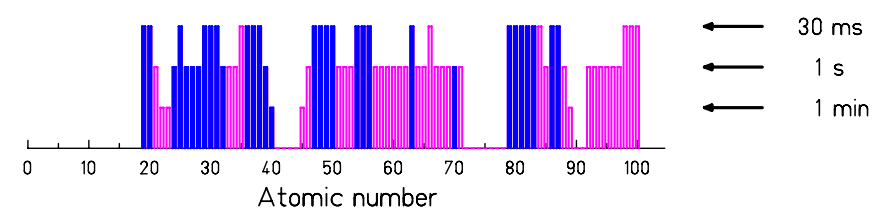

FIG. 5. (Color) Systematic overview on release-time properties of the different elements with $Z>18$. The heights of the columns denote the elements for which efficiencies of the order of $1 \%$ or more are reached for half-lives above $30 \mathrm{~ms}, 1 \mathrm{~s}$, and $1 \mathrm{~min}$, respectively. Blue columns denote those elements for which $Z$ selection is possible without laser ionization (compilation by Kirchner, 2001 [11-15]). 
The efficiency scales roughly with $\left(t_{1 / 2} / \tau\right)^{\alpha}$ for half-lives $t_{1 / 2}$, which are shorter than the corresponding time parameter $\tau$. The exponent $\alpha$ shows some variation around the mean value of about 1.5. The value of the parameter $\tau$ depends on both the element to be extracted and the material properties of the ISOL target. Typical values found for the operation of ISOLDE reach from $1 \mathrm{~s}$ to several hundreds of seconds and more.

Based on these results, one expects the overall efficiency to decrease roughly by a factor of 4 from one isotope to the next in the direction towards the drip lines, once the halflives $t_{1 / 2}$ become smaller than the time parameter $\tau$. This estimate is valid in the region around $A=100$, while the overall efficiencies vary more strongly for lighter nuclei.

Obviously, fast extraction is very important for keeping decay losses of short-lived nuclides small, since these are in most cases the most exotic and thus the most interesting species, for which already the in-target production rates decrease steeply when approaching the drip lines. But also alternative, technically more complex solutions, which avoid the decay losses, might be envisaged. In the design of the RIA facility, projectile-fragmentation reactions induced by stable nuclear beams and in-flight separation as well as the use of a gas catcher have been considered to overcome this problem, see, e.g., Ref. [17]. As already mentioned, for the EURISOL project, the fragmentation and in-flight separation of a reaccelerated neutron-rich ISOL beam [10] is being investigated, which should allow producing extremely neutron-rich nuclides. However, a detailed discussion of this option is beyond the scope of the present paper.

\section{Nuclear-reaction aspects}

Different types of nuclear reactions are exploited for the nuclide production in the baseline design of the EURISOL project, driven by a $1-\mathrm{GeV}$ proton beam. In the directtarget option, spallation-evaporation and spallation-fission residues are produced in mostly direct interactions of the primary protons with different target material. In the highpower fission-target option, secondary neutrons from a converter target induce fission in a uranium or thorium target at excitation energies mostly up to a few $\mathrm{MeV}$ above the fission barrier. Neutron-induced fission was also considered as one of the neutron-rich nuclide production mechanisms for RIA, and some estimates of possible beam intensities can be found in [17].

From recent experiments performed at GSI in inverse kinematics, there is good empirical knowledge on the general characteristics of these reactions. In addition, the development of realistic nuclear-reaction codes has strongly advanced on the basis of these new data.

Figures 6-8 present the measured nuclide distributions for $1 \mathrm{GeV}$ proton-induced reactions on ${ }^{56} \mathrm{Fe},{ }^{136} \mathrm{Xe}$, and ${ }^{238} \mathrm{U}$, respectively, measured in inverse kinematics [1824]. In addition, the nuclide distribution produced in fission

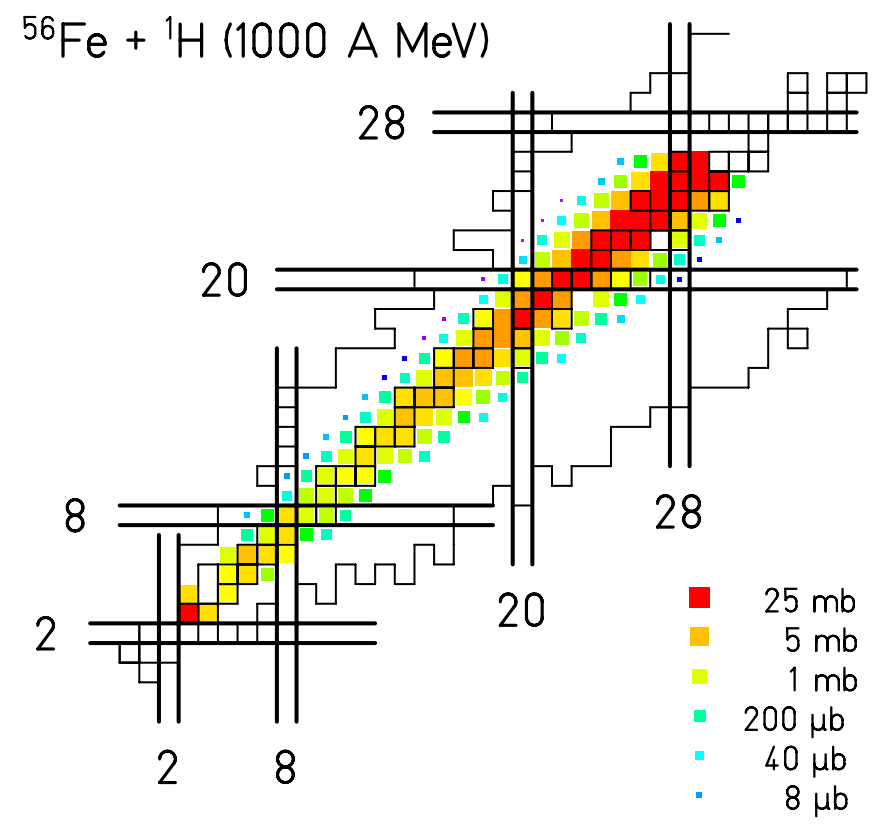

FIG. 6. (Color) Measured nuclide distribution from the reaction ${ }^{56} \mathrm{Fe}(1 A \mathrm{GeV})+{ }^{1} \mathrm{H}[18,19]$.

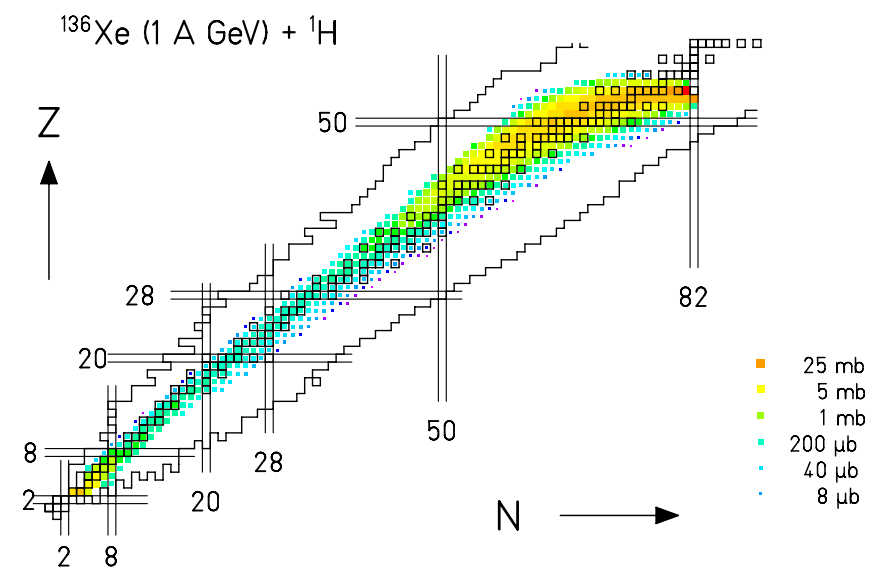

FIG. 7. (Color) Measured nuclide distribution from the reaction ${ }^{136} \mathrm{Xe}(1 A \mathrm{GeV})+{ }^{1} \mathrm{H}[20]$.

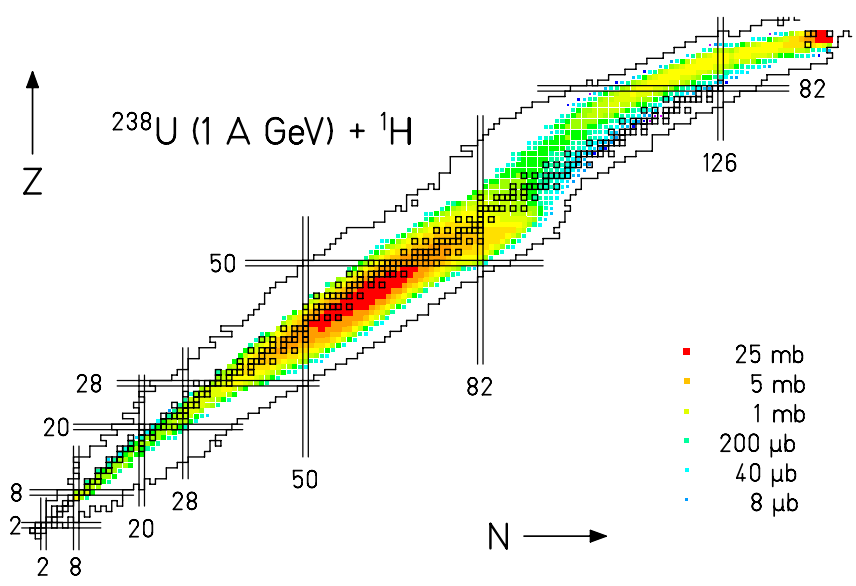

FIG. 8. (Color) Measured nuclide distribution from the reaction ${ }^{238} \mathrm{U}(1 A \mathrm{GeV})+{ }^{1} \mathrm{H}[21-24]$. 
of ${ }^{239} \mathrm{U}$ at the typical excitation energy of $2 \mathrm{MeV}$ above the fission barrier (corresponding to the fission of ${ }^{238} \mathrm{U}$ induced by $3.5 \mathrm{MeV}$ neutrons) is shown in Fig. 9 as calculated with the ABRABLA code [25-28].

This overview reveals the following general characteristics: (i) Spallation-evaporation residues populate neutron-rich isotopes of a few elements below the target ${ }^{2}$ ) with strongly decreasing cross sections by coldfragmentation reactions [29]. This is an option for the production of neutron-rich isotopes above the fission region. However, the limited choice of target elements imposes a severe restriction on this option. (ii) The main nuclide production by spallation-evaporation reactions gradually tends towards the proton-rich side and extends to roughly 10 to 15 elements below the target element. Saturation in the production of the most neutron-deficient isotopes is reached around 5 elements below the target, where the cross sections already decrease. The limited choice of target elements imposes again some restriction on this option to produce isotopes at the proton drip line. Lighter residues are situated closer to the beta-stability line. (iii) Spallation-fission reactions with actinide targets (uranium and thorium are technical options) produce neutron-rich isotopes in a wide element range (extending from about $Z=10$ to $Z=60$ and further) with high cross sections, see Fig. 8. (iv) Spallation-fission reactions with lighter targets (e.g. lead, see Fig. 11) are not interesting for the production of radioactive beams, because they produce nuclides rather close to the beta-stability line. Moreover, the cross sections are rather low. (v) The nuclide production by fission of ${ }^{238} \mathrm{U}$ target induced by neutrons of a few $\mathrm{MeV}$ is focused on about 8 very neutron-rich isotopes of a limited number of elements between $Z \approx 30$ to $Z \approx 62$ with a dip around symmetric fission $(Z \approx 46)$, see Fig. 9 .

These characteristics were already qualitatively known before and were exploited, e.g., for the production of radioactive species at the ISOLDE facility. However, the new experimental results and the improved codes provide a better quantitative and systematic knowledge, which is important for the prediction of yields.

The high-power target option deserves some additional remarks: Because of the deposition of the primary-beam energy in the converter target and the concentration of the production to a very limited number of nuclides, one obtains very high in-target production rates. Best conditions for very high secondary-beam intensities by the ISOL method are provided for nuclides around ${ }^{132} \mathrm{Sn}$. The double shell closure is responsible for several peculiarities: (i) High production yields in the heavy fission-fragment group go in line with an enhancement of the $N / Z$ ratio due to strong charge polarization, see Fig. 9. (ii) Long half-lives

\footnotetext{
${ }^{2}$ Although the experiment was performed in inverse kinematics, we refer here to normal kinematics, which is applied in the EURISOL scenario. Therefore, the heavy reaction partner is denoted as the target nucleus.
}

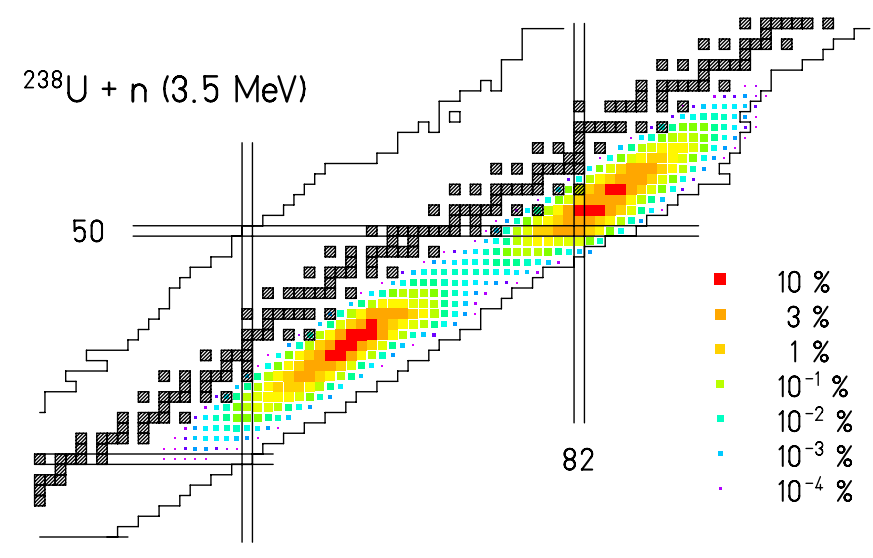

FIG. 9. (Color) Nuclide distribution of fission products from the neutron-induced fission of ${ }^{238} \mathrm{U}$ for fixed neutron energy of $E_{n}=$ $3.5 \mathrm{MeV}$, calculated with the nuclear-reaction code ABRABLA. The color code gives the yields in percent.

extend to rather large values of the neutron excess, see Fig. 4.

Luckily, the mostly produced elements of the heavy fission-fragment group (tin to lanthanum) can be extracted from the production target with rather high efficiencies by the ISOL method. In addition, their long half-lives allow for using rather large target volumes, which are connected with long extraction times, without introducing excessive decay losses.

The nuclides in the light fission-fragment group are less favored: First, they are less neutron-rich; second, they have shorter half-lives. However, also here the elements from zinc $(Z=30)$ to strontium $(Z=38)$ can be extracted from the production target with rather high efficiencies by the ISOL method. However, moderate target volumes should be used in order to limit the decay losses.

\section{SOME POSSIBILITIES TO ENHANCE THE PRODUCTION YIELDS FOR SPECIFIC NUCLIDES}

In the course of the intense discussion on the design of future secondary-beam facilities, different scenarios for the production of secondary beams have been introduced. In particular, numerous ideas have been developed, which exploit specific aspects of nuclear reactions and technical solutions, e.g., with respect to limitations on heat load, ISOL efficiencies, and background intensities. The following sections present, in a qualitative way, the basic ideas of several of these propositions, which seem to be promising for the EURISOL facility. The assessment of the quantitative benefit in comparison with the standard $1 \mathrm{GeV}$ protondriver option of the EURISOL project will be given in Sec. V.

\section{A. Extending the mass range of spallation-evaporation residues by a $2 \mathrm{GeV}$ primary beam}

It has been observed that the intensities of several nuclides increase strongly if the energy of the primary beam 
grows over a certain range. Figure 10 demonstrates this behavior.

A better understanding of the nuclear-reaction characteristics, which are responsible for this phenomenon, can be obtained on the basis of systematic measurements on the nuclide production induced by $500 \mathrm{MeV}$ and $1 \mathrm{GeV}$ protons as well as $2 \mathrm{GeV}$ deuterons on ${ }^{208} \mathrm{~Pb}$ [31-34], recently performed in inverse-kinematics experiments at GSI, Darmstadt. In all three cases, which are shown in Fig. 11, the nuclide distributions consist of two components. Spallation followed by evaporation populates the heavy component, which extends from the projectile to lighter neutron-deficient nuclei. Spallation followed by a fission process (in addition to evaporation before and after fission) populates another region close to beta stability, centered somewhat below one- half the mass of the projectile.

We can observe that the spallation-evaporation distribution extends to lighter masses, when the center-of-mass energy of the system is increased, while the spallationfission distribution is less sensitive to the energy. For a center-of-mass energy of about $500 \mathrm{MeV}$, the spallationevaporation distribution extends over essentially 5 elements, for about $1 \mathrm{GeV}$, essentially 10 elements are formed, while for about $2 \mathrm{GeV}$ more than 15 elements are formed. Thus, the increase in the cross sections shown in Fig. 10 originates from the extension of the spallation-

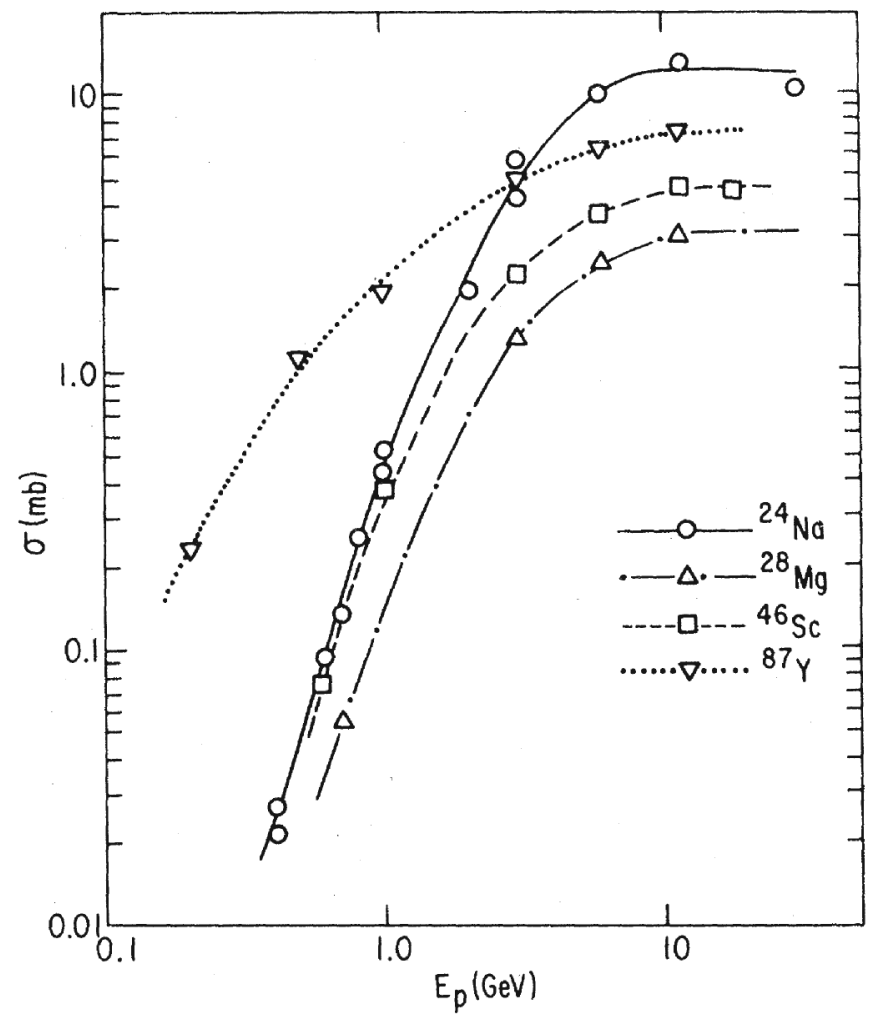

FIG. 10. Measured excitation function for the fragmentation of gold by protons leading to some typical light products (taken from Ref. [30]).

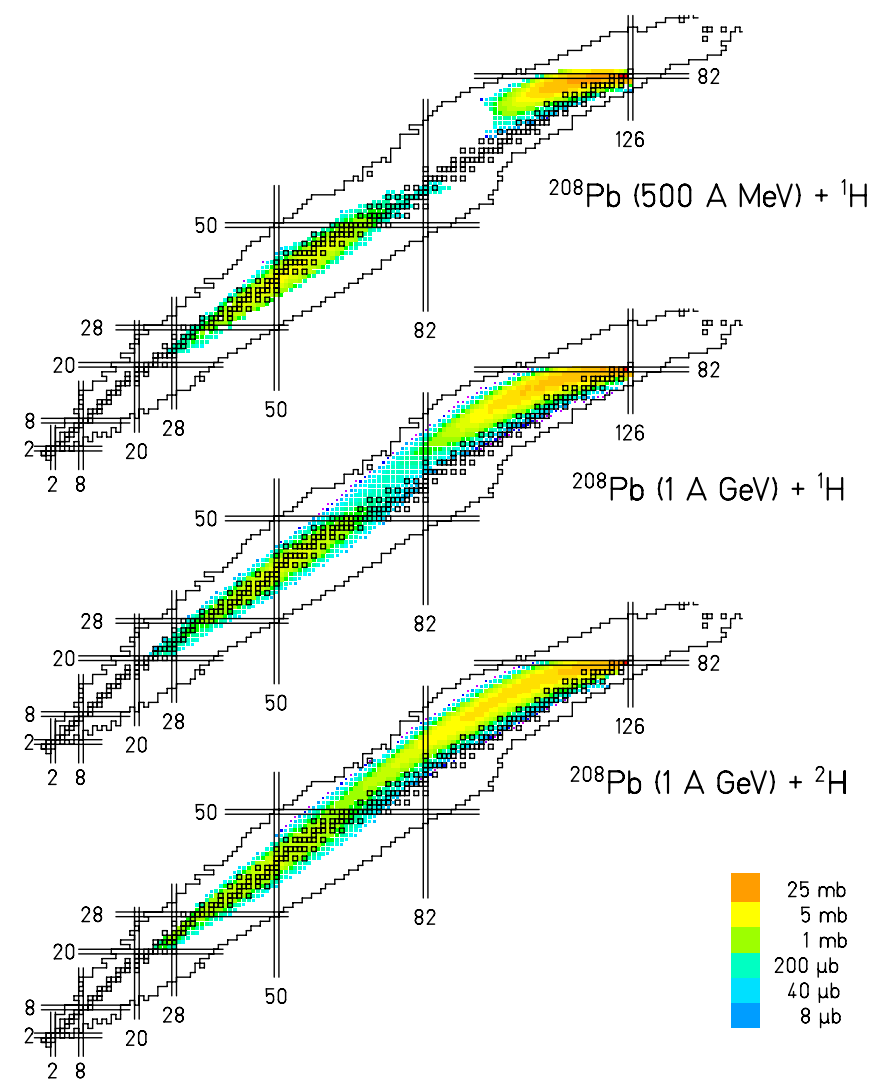

FIG. 11. (Color) Nuclide distributions of residues produced in the spallation of ${ }^{208} \mathrm{~Pb}$ by $500 \mathrm{MeV}$ protons, $1 \mathrm{GeV}$ protons, and $2 \mathrm{GeV}$ deuterons, measured at GSI in inverse kinematics [3134]. Elements below $Z \approx 20$ were not covered in the experiments.

evaporation distribution to lighter masses with higher energy.

From this observation, one may conclude that the most neutron-deficient isotopes of all elements that are available by ISOL techniques can be produced with high intensities, if the beam energy is chosen sufficiently high to bridge the gaps between the different target materials. A quantitative discussion of this aspect will follow in Sec. VA.

\section{B. Enhancing intermediate-mass fragment (IMF) emission to produce light neutron-rich nuclides by a $2 \mathrm{GeV}$ primary beam in a fissile target}

While the total fission cross section stays almost the same when the beam energy is increased form 1 to $2 \mathrm{GeV}$, the production cross section of light binary products below $Z=30$ is rather sensitive to the energy deposited in the uranium nucleus. This is clearly seen in some measurements performed at ISOLDE. Figure 12 represents production cross sections of sodium isotopes in uranium at 1.0 and $1.4 \mathrm{GeV}$ proton energy in a uranium-carbide target, deduced from detailed measurements of release curves at ISOLDE [36,37]. Production cross sections for ${ }^{21,24,25} \mathrm{Na}$ by $600 \mathrm{MeV}$ protons in uranium are also shown. These 


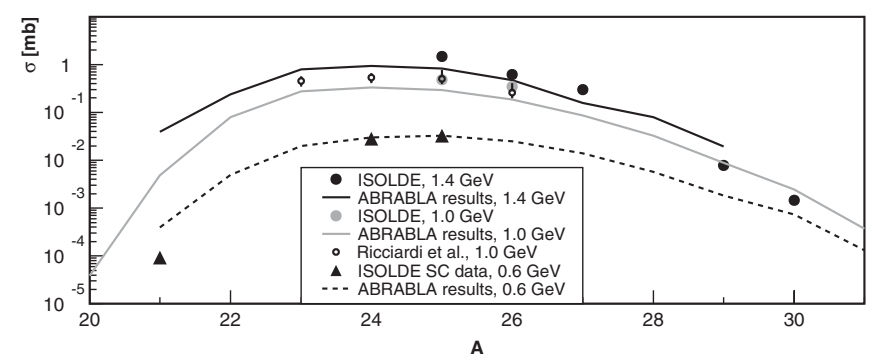

FIG. 12. $\mathrm{Na}$ isotopic production cross sections in uranium. Results deduced from release measurements at ISOLDE at 0.6, 1.0 , and $1.4 \mathrm{GeV}$ proton energies are included, along with ABRABLA calculations at the same energies, and results by Ricciardi et al. measured in inverse kinematics at GSIDarmstadt [35].

have been deduced from the ISOLDE SC final yield data $[2,38]$ and not from the detailed release curves, so that it was impossible to correct for the decay losses. However, $21,24,25 \mathrm{Na}$ are sufficiently long lived that their decay losses can be neglected (typically $1 \%-5 \%$ for ${ }^{21,25} \mathrm{Na}$, and even less for ${ }^{24} \mathrm{Na}$ ).

For comparison, production cross sections of sodium isotopes obtained by the ABRABLA code are also shown. The energy dependence of the deduced cross sections is clearly seen, which confirms the ABRABLA predictions. Thus, an important gain in cross section for nuclei in this mass range in a fissile target is to be expected, if the energy of the primary beam is increased.

\section{Extended fission-fragment distribution in neutron- induced fission using deuteron-converter neutron source, with $E_{n}=20$ to $80 \mathrm{MeV}$}

For the EURISOL facility, a high-power converter target is being designed. It consists of several essentially cylindrical parts, see Fig. 13. The inner cylinder is the converter, which acts as a spallation neutron source, converting the $1 \mathrm{GeV}$ proton beam into a neutron flux. The converter is surrounded by the production target of a fissile material, where the neutrons induce fission reactions that produce the desired neutron-rich nuclides.

The neutron flux in the production target is dominated by evaporative neutrons with a mean energy of about $2 \mathrm{MeV}$ as shown in Fig. 14.

Because of these low neutron energies, the fission process is induced at energies rather close to the fission barrier, leading to fission-fragment distributions, which are strongly influenced by shell effects and which are dominated by asymmetric fission as demonstrated in Fig. 9. The production concentrates on very neutron-rich isotopes of a few elements around $\mathrm{Xe}(Z=54)$ and $\operatorname{Sr}(Z=38)$. The elements between $Z=42$ and $Z=48$ are only weakly produced, and the distribution dies out for $Z<32$ and $Z>60$.

Because of the high neutron-capture cross section of ${ }^{235} \mathrm{U}$ for low neutron energies, more than half of the nuclide

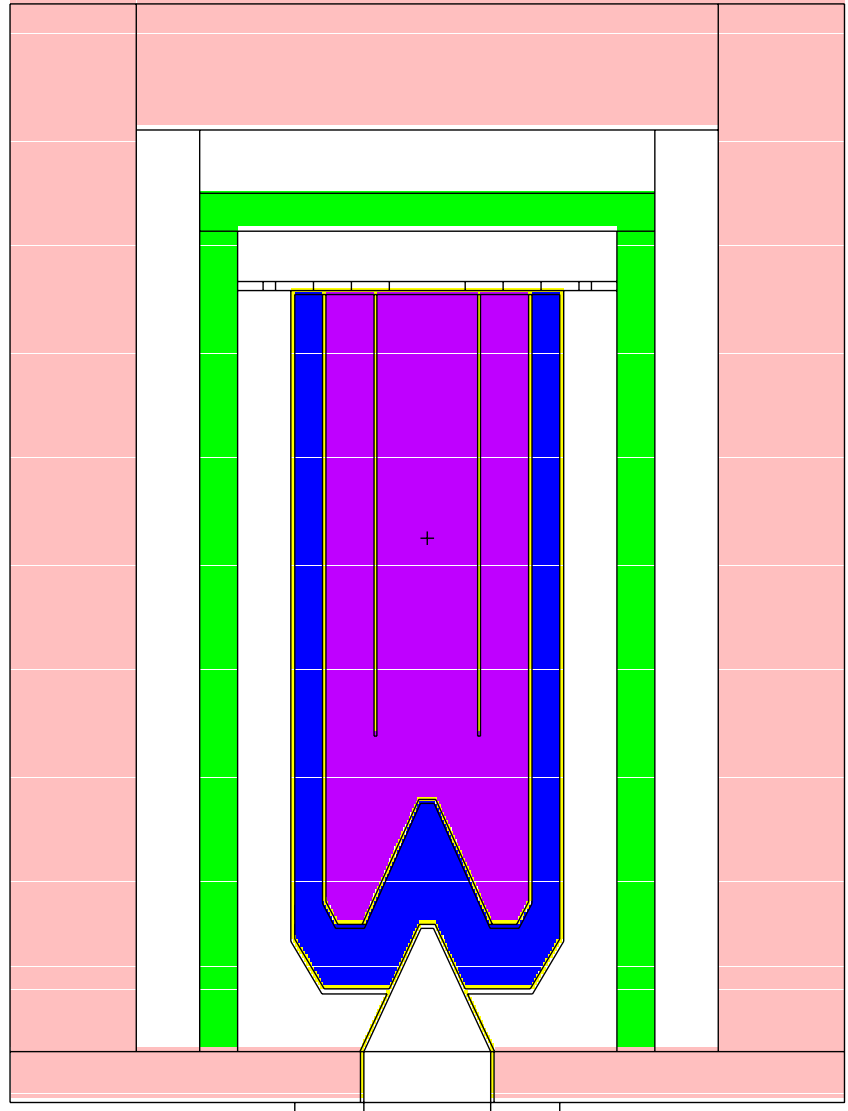

FIG. 13. (Color) A possible design of the high-power converter target for EURISOL. The drawing shows a cut through the cylindrical assembly. The violet area in the center denotes the mercury converter, which is hit by the $1 \mathrm{GeV}$ proton beam, entering from the lower side. The green areas mark the uranium production target [39].

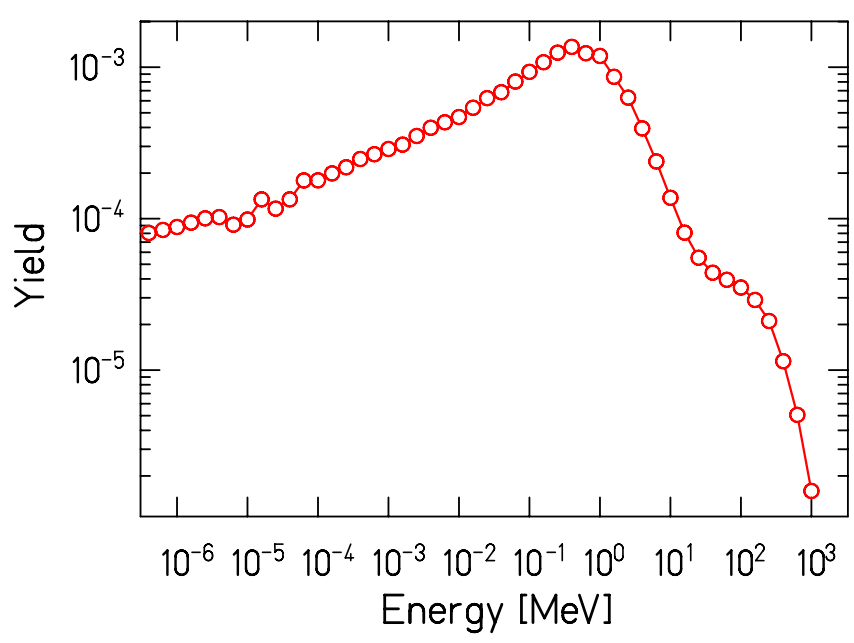

FIG. 14. (Color) Calculated energy distribution of the neutron flux around the converter of the EURISOL high-power target [39]. 


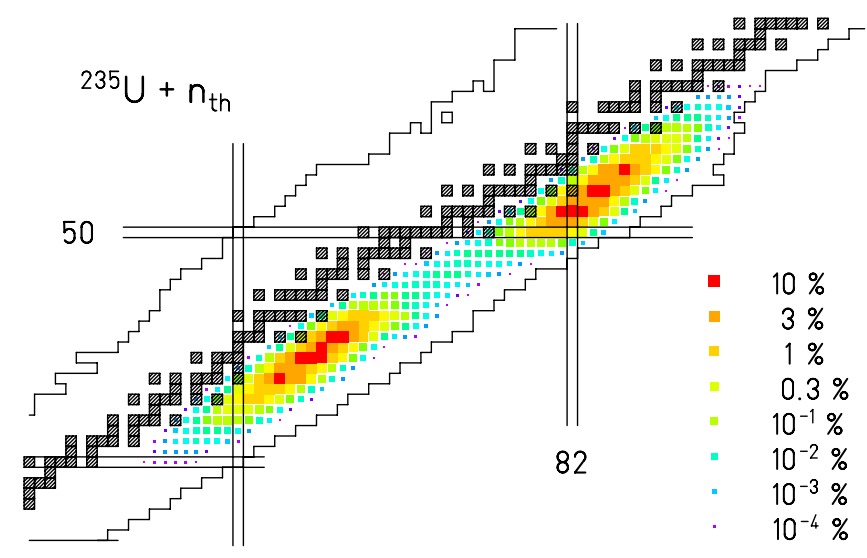

FIG. 15. (Color) Nuclide distribution of fission products from thermal-neutron-induced fission of ${ }^{235} \mathrm{U}$, calculated with the nuclear-reaction code ABRABLA. The color code gives the yields in percent.

production in a production target of natural uranium is due to fission of the compound nucleus ${ }^{236} U$ [39]. As can be seen in Fig. 15, the nuclide distribution is slightly shifted to the neutron-deficient side if compared to low-energy neutron-induced fission of ${ }^{238} \mathrm{U}$, see Fig. 9. The difference of 3 neutrons in the fissioning nucleus leads to an average loss of about 1.5 neutrons in the fission fragments. Thus, the neutron-induced fission of ${ }^{235} \mathrm{U}$ is less favorable for producing the most neutron-rich fission fragments. The total production in the high-power target is roughly given by the superposition of the nuclide distributions of Figs. 9 and 15 .

As the total fission rate is limited from the point of view of the target handling, it would be advantageous to use depleted ${ }^{238} \mathrm{U}$ for the target in order to enhance the production of more neutron-rich nuclides.

It has been discussed in the planning for RIA and it has become the standard option for SPIRAL 2 to apply a different converter scheme using deuterons as a primary beam, leading to a neutron flux of appreciably higher energies. The resulting energy spectrum of the secondary neutrons peaks a bit below $\frac{1}{2}$ of the initial deuteron energy. That means that the neutrons essentially keep the velocity of the primary beam at the place in the target where they are produced. In addition, they are mostly forward directed. This is demonstrated in Fig. 16 for a primary beam energy of $50 \mathrm{MeV}$. Because of the condition that the deuterons should be stopped in the converter target and, at the same time, most of the neutrons should leave the converter target without being absorbed, the beam energy of this approach is limited to about $200 \mathrm{MeV}$. At SPIRAL 2 , a primary energy of only $40 \mathrm{MeV}$ will be used in order to limit the influence of neutron evaporation from the fragments, which tends to reduce the neutron excess of the fission products.

The influence of the higher neutron energy on the nuclide distribution for the neutron-induced fission of ${ }^{238} \mathrm{U}$

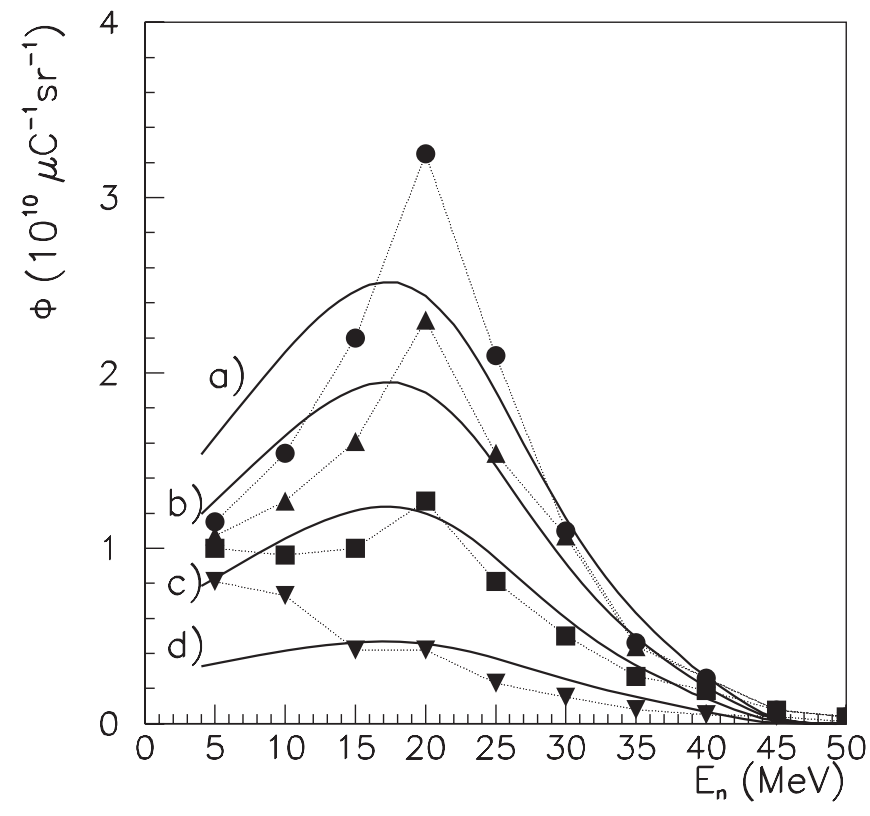

FIG. 16. Experimental energetic distributions of neutrons for $50 \mathrm{MeV} 2 \mathrm{H}$ incident energy on a thick Be target: filled circles at $0^{\circ}$, filled triangles with up-point at $5^{\circ}$, filled squares at $10^{\circ}$, and filled triangles with down-point at $25^{\circ}$. These distributions are compared with theoretical ones: (a) $0^{\circ}$, (b) $5^{\circ}$, (c) $10^{\circ}$, and (d) $25^{\circ}$. The dotted lines are to guide the eye. The figure is taken from Ref. [40].

can be seen by comparing Fig. 17, which shows the production in ${ }^{238} \mathrm{U}+20 \mathrm{MeV}$ neutrons, with Fig. 9. Three tendencies can be observed: Symmetric fission is more abundant, populating the elements between $Z=42$ and $Z=48$ much more strongly, the distribution is slightly shifted to less neutron-rich isotopes, and the fissionfragment distribution extends slightly further to the heavier and to the lighter masses. As a consequence, the production of nuclei in the vicinity of ${ }^{78} \mathrm{Ni}$ seems to be slightly

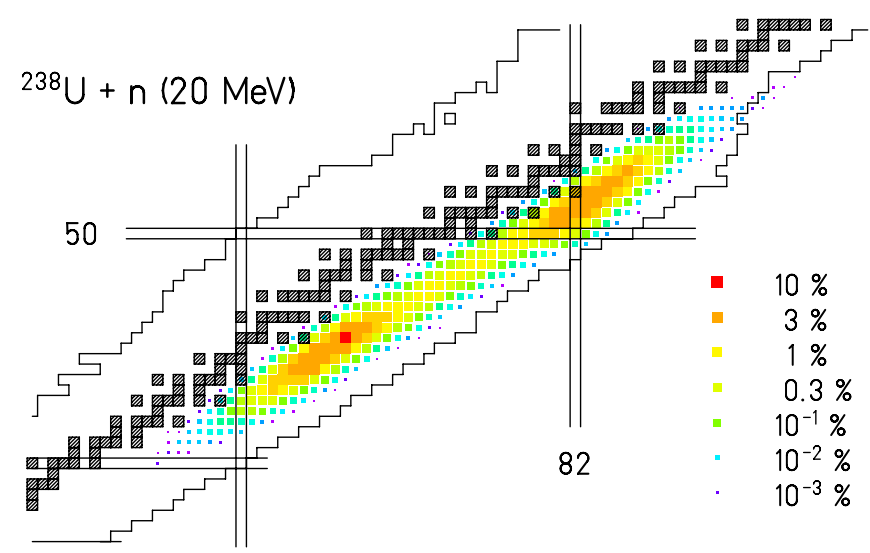

FIG. 17. (Color) Nuclide distribution of fission products from the neutron-induced fission of ${ }^{238} \mathrm{U}$ for a fixed neutron energy of $E_{n}=20 \mathrm{MeV}$, calculated with the nuclear-reaction code ABRABLA. The color code gives the yields in percent. 


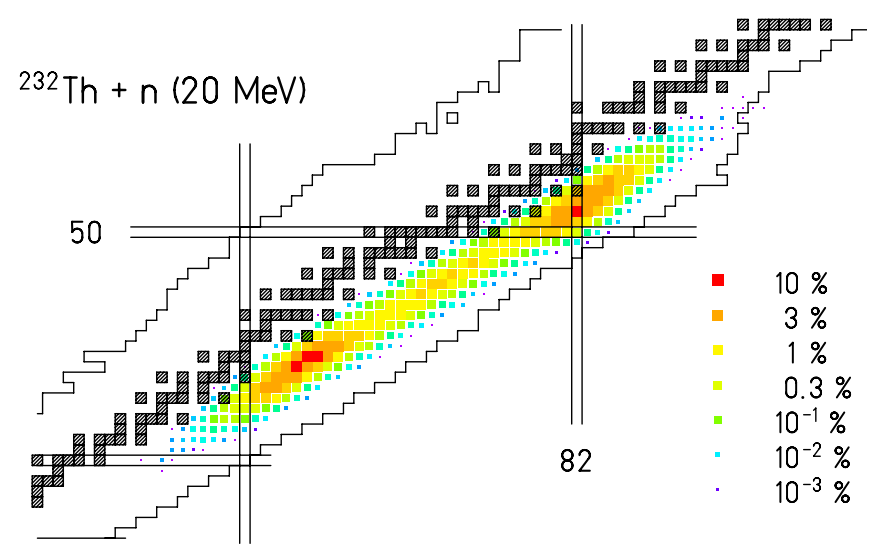

FIG. 18. (Color) Nuclide distribution of fission products from the neutron-induced fission of 232Th for a fixed neutron energy of $E_{n}=20 \mathrm{MeV}$, calculated with the nuclear-reaction code ABRABLA. The color code gives the yields in percent.

enhanced. The loss in the production of very neutron-rich isotopes is not strongly pronounced.

If ${ }^{232} \mathrm{Th}$ is used as target material in the deuteronconverter option, the neutron excess of the fission fragments is expected to be reduced by about 0.4 units only. However, the light peak of the fission fragments moves to lighter masses by about 6 units. The calculated nuclide production for this option is shown in Fig. 18. This may be another efficient way to enhance the production of nuclides in the vicinity of ${ }^{78} \mathrm{Ni}$. These conclusions are consistent with experimental results on fission-fragment mass distributions in proton-induced reactions with ${ }^{232} \mathrm{Th}$ and ${ }^{238} \mathrm{U}$ targets [41].

Quantitative considerations on these different options will be presented in Sec. V C.

\section{Exploiting general properties of heavy-ion fragmentation reactions}

It has been observed that the use of heavy ions instead of proton projectiles in irradiation of a uranium-carbide target gives enhanced yields per incident ion and broader isotopic distributions for light elements. This finding is demonstrated in Fig. 19, which is taken from Refs. [42,43]. It seems that the production of long-lived isotopes close to beta stability is enhanced by a factor of 2 and 5 by ${ }^{3} \mathrm{H}$ and ${ }^{12} \mathrm{C}$ projectiles, respectively. As already discussed by Ravn et al. [3], the enhancement factor increases for the more neutron-rich isotopes. However, one should not forget that at the same time these are characterized by shorter life times. Thus, one may be cautious about concluding on the origin of the enhanced yields.

It is interesting that the pattern in the enhancement factor observed for the isotopes ${ }^{28} \mathrm{Na}\left(t_{1 / 2}=30.5 \mathrm{~ms}\right)$, ${ }^{29} \mathrm{Na}\left(t_{1 / 2}=44.9 \mathrm{~ms}\right),{ }^{30} \mathrm{Na}\left(t_{1 / 2}=48 \mathrm{~ms}\right)$, and ${ }^{31} \mathrm{Na}$ $\left(t_{1 / 2}=17 \mathrm{~ms}\right)$ does not scale with the mass number but rather with the inverse of the half-life. Indeed, Fig. 20
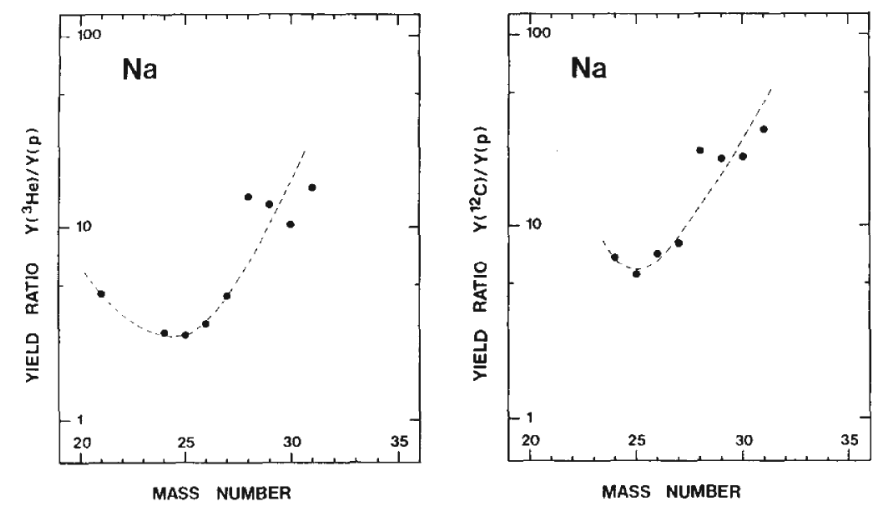

FIG. 19. Ratio of sodium beam yields per incident ion obtained by fragmentation of uranium by means of $300 \mathrm{~A} \mathrm{MeV}{ }^{3} \mathrm{He}$ and $78 \mathrm{~A} \mathrm{MeV}{ }^{12} \mathrm{C}$ over fragmentation of uranium by means of $600 \mathrm{MeV}$ protons (figure taken from Ref. [3]).

illustrates that the production ratios correlate with the half-life rather well, in particular, for the short-lived isotopes.

In this context, we would like to mention that, from the operation of ISOLDE, a decay loss of more than a factor of hundred was deduced for ${ }^{28} \mathrm{Na}$ [16]. Thus, we are inclined to suppose that at least part of the enhancement and most of its variation with mass number is caused by smaller decay losses in the heavy-ion-induced reactions. The overall efficiencies for the long-lived isotopes seem also to be correlated with the different ranges of $600 \mathrm{MeV}$ protons $\left(321 \mathrm{~g} / \mathrm{cm}^{2}\right), 297 A \mathrm{MeV}^{3} \mathrm{He}\left(82.2 \mathrm{~g} / \mathrm{cm}^{2}\right)$, and $78 A \mathrm{MeV}$ ${ }^{12} \mathrm{C}\left(3.8 \mathrm{~g} / \mathrm{cm}^{2}\right)$ in uranium, which can be compared to the

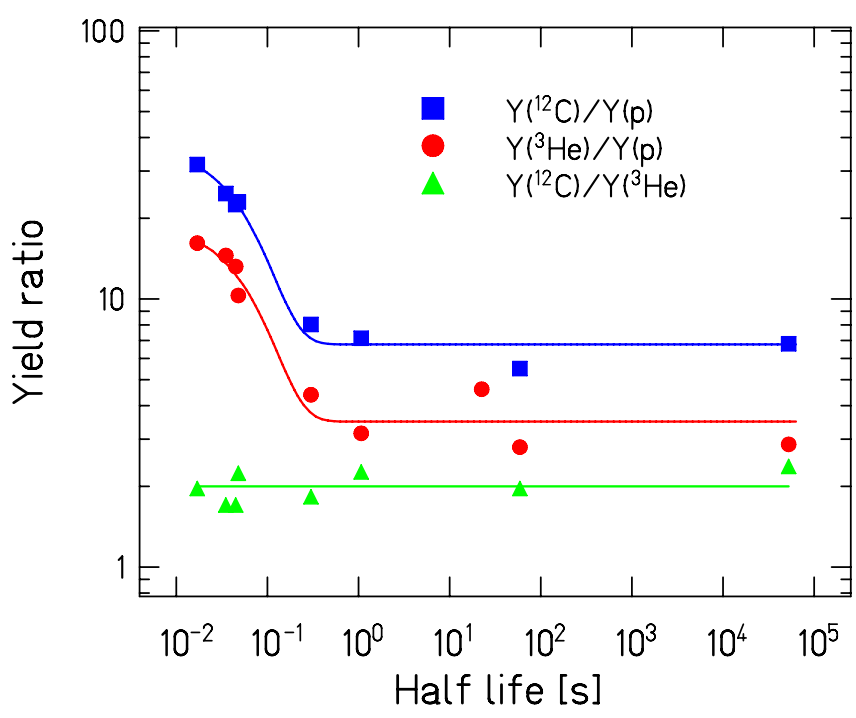

FIG. 20. (Color) Measured yield ratios of different sodium isotopes obtained by fragmentation of uranium by $300 A \mathrm{MeV}{ }^{3} \mathrm{He}$ (dots) and $78 \mathrm{~A} \mathrm{MeV}{ }^{12} \mathrm{C}$ (squares) over spallation of uranium by $600 \mathrm{MeV}$ protons as a function of the isotope half-life. The ratio between ${ }^{12} \mathrm{C}$ - and ${ }^{3} \mathrm{He}$-induced fragmentation (triangles) is also shown. 
target thickness of $12 \mathrm{~g} / \mathrm{cm}^{2}$. (The range in carbon, which also is a constituent of the production target, scales about in the same way.) A similar correlation can be found with the specific energy-loss values in $1 \mathrm{mg} / \mathrm{cm}^{2}$ uranium: $1.3 \mathrm{keV}$ for $600 \mathrm{MeV}$ protons, $6.8 \mathrm{keV}$ for $297 \mathrm{~A} \mathrm{MeV}{ }^{3} \mathrm{He}$, and $136 \mathrm{keV}$ for $78 \mathrm{~A} \mathrm{MeV}{ }^{12} \mathrm{C}$.

The actual physical reason for the presumed variation of the decay losses remains unclear to us. We may speculate that small differences in the parameters of the experiment, e.g., in the target temperature or in the beam position, might have caused slightly different overall-efficiency values and, in particular, different decay losses. In any case, it seems to us that such kind of differential measurements is a very demanding task.

From the given data set, it is difficult to draw a definite conclusion on the magnitude of the cross-section increase for the short-lived isotopes. The higher yields for longlived isotopes, however, might partly be related to an enhanced emission of intermediate-mass fragments in the case of heavier projectiles, which will be discussed in Sec. IV F.

\section{E. Converter-catcher-ISOL scenario using heavy-ion beams}

In the contribution of the target-and-ion-source group (Appendix C, Sec. 2.2.3) to the EURISOL RTD report [4], the use of an intense heavy-ion beam impinging on a beampower-dissipating target of lithium or carbon, followed by a fragment catcher-diffuser, was proposed as an alternative option for the production of light exotic beams. This approach should offer several important advantages.

The first advantage of using heavy ions stems from the possibility of using different beams - including isotopically enriched projectiles - that can be optimally chosen. In this way, one could overcome the problems due to restrictions on the target material discussed in Sec. III A. The choice of a light heavy-ion beam can provide the opportunity to use nuclei which are in principle not available as target material, e.g., gaseous elements such as, e.g., oxygen or noble gases. An example of such approach is the use of an ${ }^{18} \mathrm{O}$ beam for the production of ${ }^{14} \mathrm{Be}$. In case of oxygen, there is a principal possibility to use an oxide target; however beryllium is not released from such target material. Moreover, such a target would require a large quantity of the separated ${ }^{18} \mathrm{O}$, which is very expensive. A similar possibility is the use of a neon beam for the production of the nuclide ${ }^{18} \mathrm{Ne}$ which is of interest for the beta-beam capability of EURISOL. A quantitative comparison for both cases will be carried out in Sec. VE.

Another important aspect is that for the production of nonfission fragments, this option also allows for splitting the target into two parts, with distinct functions, i.e., firstly a primary-to-secondary beam "converter" target and secondly, a "catcher" for the effusion-diffusion of the stopped secondary ions. Thus, the functions of the target for pro-

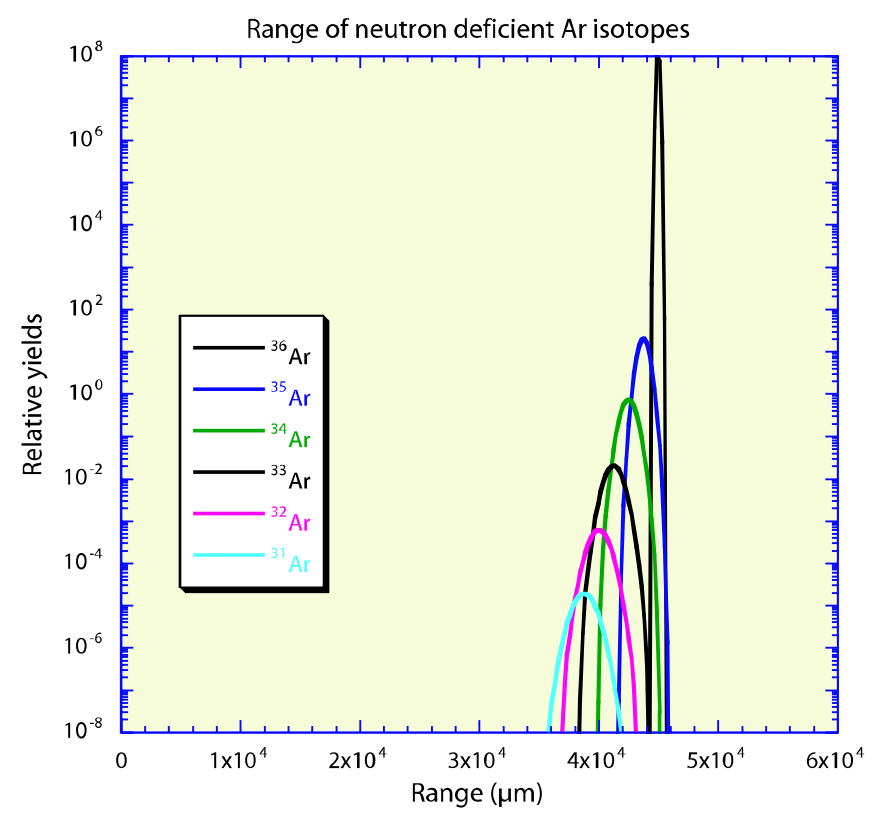

FIG. 21. (Color) Range distribution of the projectile and neutron-deficient argon isotopes produced by fragmentation of a ${ }^{36} \mathrm{Ar}$ projectile at $333 \mathrm{~A} \mathrm{MeV}$. The decoupling of production target, diffusion catcher, and beam stopper is possible even in the case of neutron-deficient reaction products, so that most of the beam energy is deposited in the stopper. The figure is taken from Ref. [4].

duction and diffusion are separated, allowing one to choose the most resilient diffusion-catcher in most cases. Owing to the narrow forward emission cone of the fragments, the beam-power-dissipater/target may more easily be dissociated from the diffusion catcher, and (because of their shorter range) this allows one to use a more compact catcher structure that enhances the effusion speed. The technique of a two-step target is already in use at the present SPIRAL facility for the production of ${ }^{6,8} \mathrm{He}$ isotopes in the reaction ${ }^{13} \mathrm{C}+\mathrm{C}$. In this case, the first part stops the primary ${ }^{13} \mathrm{C}$ beam, and simultaneously produces ${ }^{6,8} \mathrm{He}$ by projectile fragmentation. The second part stops the projectile fragments of ${ }^{6,8} \mathrm{He}$. But also for neutrondeficient reaction products, the decoupling of production target, diffusion catcher, and beam stop is possible, as demonstrated in Fig. 21. In this way, the respective optimum materials can be used for these different layers. A more quantitative discussion is given in Sec. VE.

\section{F. Production of neutron-rich nuclides in heavy-ion reactions in the Fermi-energy regime related to nucleon exchange between target and projectile}

Nucleus-nucleus collisions in the Fermi-energy domain exhibit a large variety of contributing reaction mechanisms and reaction products (see e.g. [44-47]) and in principle offer the possibility to produce midheavy to heavy neutronrich nuclei in very peripheral collisions. An enhancement of yields of neutron-rich nuclei was observed in the reac- 

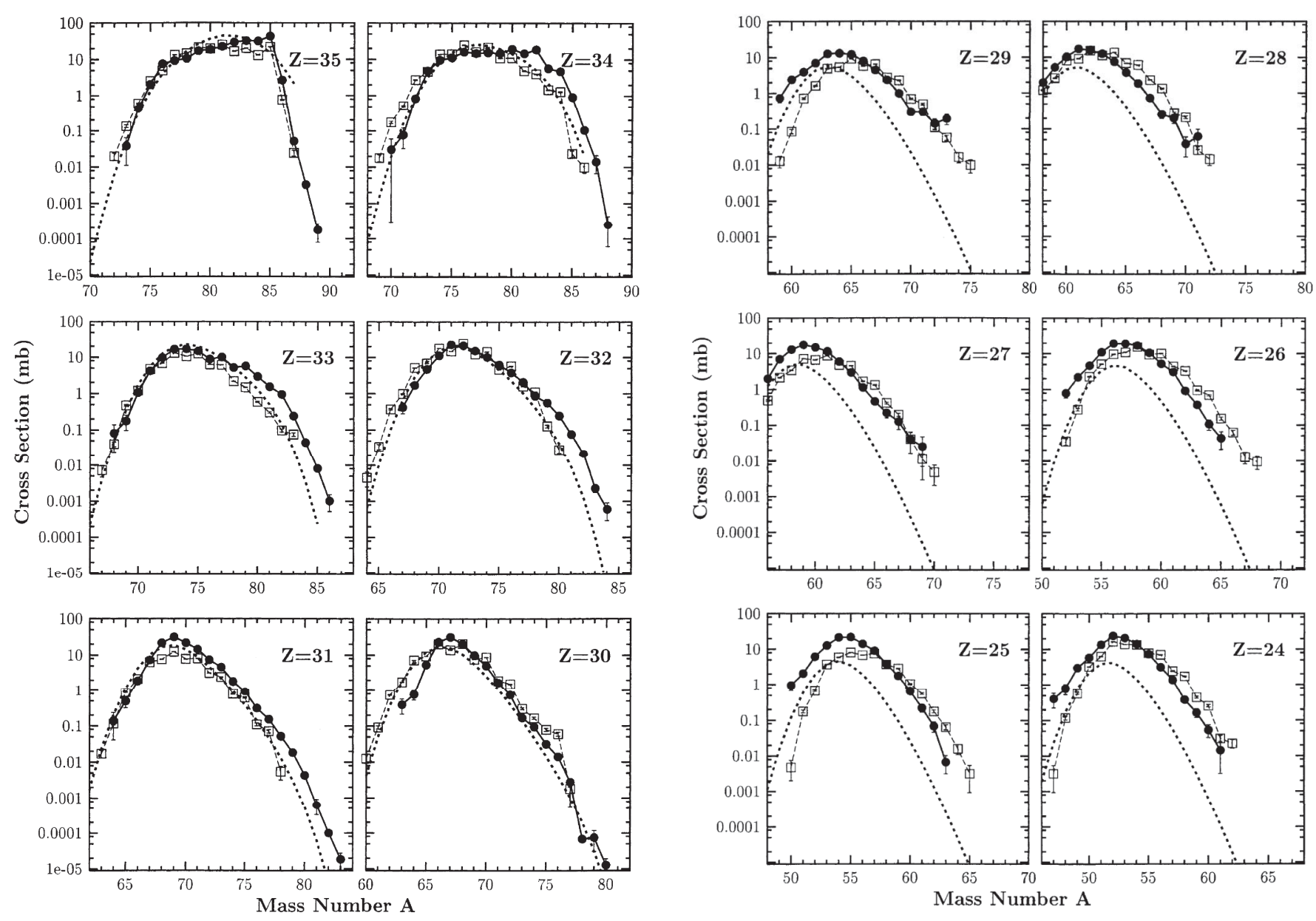

FIG. 22. Observed excess of neutron-rich nuclei in the reaction ${ }^{86} \mathrm{Kr}+{ }^{64} \mathrm{Ni}$ at $25 \mathrm{~A} \mathrm{MeV} \mathrm{[45].} \mathrm{Solid} \mathrm{symbols,} \mathrm{experimental}$ data; open symbols, DIT + Gemini. EPAX results for the same reaction are also included for comparison (dotted line).

tion ${ }^{86} \mathrm{Kr}+{ }^{64} \mathrm{Ni}[45]$ in very peripheral collisions (see Fig. $22^{3}$ ), thus pointing to the possible importance of neutron and proton density profiles at the projectile and target surfaces. In the reactions of massive heavy ions such as ${ }^{86} \mathrm{Kr}+{ }^{124} \mathrm{Sn}[48]$ and ${ }^{124} \mathrm{Sn}+{ }^{124} \mathrm{Sn}$ [49] (see Fig. 23), an enhancement was observed over the yields expected in cold fragmentation (as represented by EPAX [50] estimates), which is at present, besides fission, the method of choice to produce neutron-rich nuclei.

Peripheral nucleus-nucleus collisions can be described theoretically using the model of deep-inelastic transfer (DIT) in combination with an appropriate model of deexcitation. A very good description of experimental observables was obtained $[45,48,49,51]$ using the DIT model implementation of Tassan-Got [52] and the deexcitation codes SMM [53] and GEMINI [54]. SMM represents the

\footnotetext{
${ }^{3}$ As the EPAX formula is fitted to cross-section results at collision energies above $100 \mathrm{~A} \mathrm{MeV}$, its results presented here should be understood to refer to these higher energies.
}

FIG. 23. Observed excess of neutron-rich nuclei in reaction ${ }^{124} \mathrm{Sn}+{ }^{124} \mathrm{Sn}$ at $20 \mathrm{~A} \mathrm{MeV}$ [49]. Solid symbols, experimental data; open symbols, DIT + Gemini. EPAX results for the same reaction are also included for comparison (dotted line, see footnote to Fig. 22).

statistical model of multifragmentation (SMM) and GEMINI invokes the model of sequential binary decay. An enhancement of the yields of neutron-rich nuclei over the prediction of such calculations was observed in the reaction ${ }^{86} \mathrm{Kr}+{ }^{64} \mathrm{Ni}$ at $25 \mathrm{~A} \mathrm{MeV} \mathrm{[45].} \mathrm{The} \mathrm{shapes} \mathrm{of} \mathrm{the}$ velocity spectra suggested a process with a short time scale such as very peripheral collisions where the details of neutron and proton density profiles at the projectile and target surfaces can play a significant role. In [55], the DIT model of Tassan-Got [52] was supplemented with a phenomenological correction introducing the effect of shell structure on the nuclear periphery. A consistent agreement with experimental data is achieved using a modified DIT calculation combined with SMM in the reactions of a $25 \mathrm{~A} \mathrm{MeV}{ }^{86} \mathrm{Kr}$ beam with three different target nuclei, specifically allowing to describe the deviation of the nucleon exchange from the path toward isospin equilibration.

The success of the modified DIT calculation can be explained as a correction reflecting the modification of neutron and proton transfer probabilities in peripheral 
collisions due to the effect of shell structure on isospin asymmetry at the nuclear periphery. Such an effect is of interest for better prediction of the production rates of exotic nuclei with a wide range of $N / Z$ ratios at the new generation of rare-isotope beam facilities.

The limitation of the method is mainly in the use of relatively thin targets, and thus the method can only be effective under conditions where the losses of the in-target yield can be compensated by a corresponding increase of production cross sections. Such a situation can be typically anticipated for the most neutron-rich products, where the excess of production cross sections over the relativistic fragmentation appears to increase. Within the context of EURISOL, the reactions in the Fermi-energy domain correspond to a driver-accelerator scenario with beam energy $28 A \mathrm{MeV}$ for $A / q=3$. A complementary scenario for use of reactions in the Fermi-energy domain at EURISOL would be the acceleration of stable heavy-ion beams at the planned EURISOL postaccelerator, which is a matter of discussion.

\section{QUANTITATIVE INVESTIGATIONS}

\section{A. Extending the mass range of spallation-evaporation residues by $2 \mathrm{GeV}$ primary beam}

In the following, the benefit of a $2 \mathrm{GeV}$ primary beam in comparison with a $1 \mathrm{GeV}$ proton beam will be investigated quantitatively. The option considered here is a $2 \mathrm{GeV}$ ${ }^{3} \mathrm{He}^{2+}$ beam, mostly because of its low $A / q=1.5$ value. The quantitative answer will be based on two criteria. The first one considers the mass range populated in a spallationevaporation reaction, while the second one focuses on the population of the most neutron-deficient isotopes. The first criterion exploits the fact that the mass range populated in a spallation-evaporation reaction grows with increasing beam energy. Thus, the beam energy should be chosen in a way that the production bridges the gaps between the different usable target materials. The second criterion is based on the observation that the nuclide production by spallation evaporation on the average shifts to the neutrondeficient side with increasing mass loss in the reaction. Thus, it might be advantageous to use a heavier target material for the production of the most neutron-deficient isotopes of a certain element.

We would like to mention that the choice of the specific target materials and the secondary products in this section is made independently of the technical conditions and the scientific interest. The case study should just show the principal tendencies which one has to face due to the relatively large gaps between different usable target materials, which typically extend up to 20 elements.

\section{Filling gaps in mass}

When the desired product is far below the target, the production cross section depends strongly on the projectile
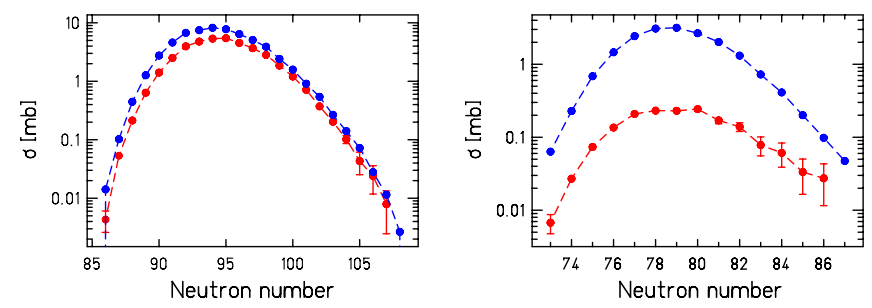

FIG. 24. (Color) Production cross sections of hafnium isotopes $(Z=72)$, left, and samarium isotopes $(Z=62)$, right, in the spallation-evaporation reaction of ${ }^{208} \mathrm{~Pb}$ with $1 \mathrm{GeV}$ protons (red symbols) and $2 \mathrm{GeV}$ deuterons (blue symbols) measured in inverse kinematics at GSI, Darmstadt.

energy. That means that higher incident energy enhances the production significantly.

Figure 24 investigates the benefit of a $2 \mathrm{GeV}$ primary beam on the production cross sections on the basis of experimental data. It shows a comparison of the measured production cross sections of hafnium $(Z=72)$ and samarium isotopes $(Z=62)$ in a lead target $(Z=82)$ by $1 \mathrm{GeV}$ protons and $2 \mathrm{GeV}$ deuterons. These elements are 10 , respectively 20, units below the target. Actually, the measurement has been made in inverse kinematics. Model calculations suggest that the nuclide production by $2 \mathrm{GeV}$ deuterons is rather similar to the one expected for $2 \mathrm{GeV}{ }^{3} \mathrm{He}$ projectiles.

Obviously, there is no benefit for the production cross sections of hafnium but a clear advantage for the production cross sections of samarium in favor of the $2-\mathrm{GeV}$ option by more than an order of magnitude. The advantage decreases for the most neutron-rich isotopes.

For a realistic estimate of the problem, the yields in a thick target with appropriate beam intensities should be considered. The variations of the beam energy due to electronic energy loss and of the intensity due to beam attenuation by nuclear reactions are depicted in Fig. 25 .

In a target of $100 \mathrm{~g} / \mathrm{cm}^{2}$, about half the projectiles interact in both cases, in $200 \mathrm{~g} / \mathrm{cm}^{2}$ the fraction is about $75 \%$. This defines a rough limit on the target thickness which is about the same for these two beams. After a $100 \mathrm{~g} / \mathrm{cm}^{2}$ target, the protons have a final energy of about $890 \mathrm{MeV},{ }^{3} \mathrm{He}$ projectiles leave the target with about $1500 \mathrm{MeV}$. The energy per projectile deposited in the same target is about a factor of 4 higher for ${ }^{3} \mathrm{He}$. This might impose a limitation on the beam intensity of ${ }^{3} \mathrm{He}$ (counted in projectile per second), which should be a factor of 4 weaker than the hydrogen beam, if the same deposited energy is considered. Considering the $100 \mathrm{~kW}(100 \mu \mathrm{A})$ proton beam according to the baseline option of EURISOL, this would imply a $50 \mathrm{~kW}(25 \mathrm{p} \mu \mathrm{A}){ }^{3} \mathrm{He}$ beam.

If the production of a specific nuclide is considered, the situation complicates further. With the energy loss after a certain distance in the target, the production of the desired element may drop severely, and any further target length becomes useless. However, the slope of the production 


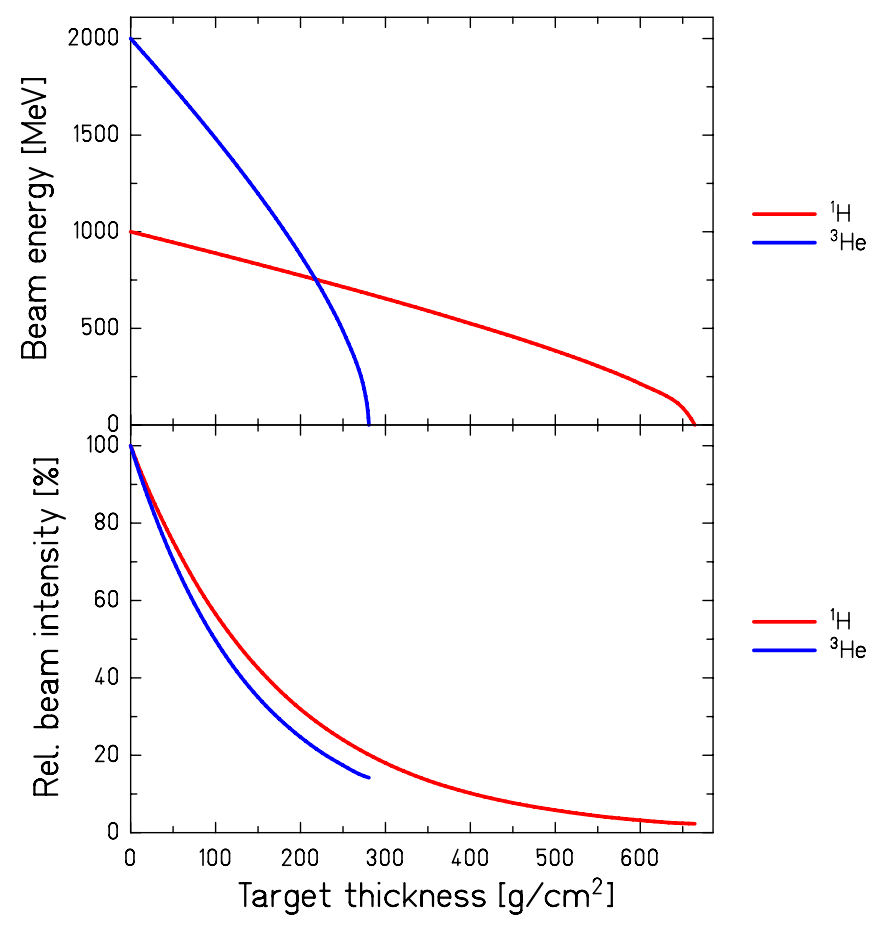

FIG. 25. (Color) Evolution of beam energy and relative intensity along a thick uranium target for a $1 \mathrm{GeV}$ proton and a $2 \mathrm{GeV}^{3} \mathrm{He}$ beam, from calculations performed with the code AMADEUS [62].

cross-section dependence on energy is higher at lower energies, and enters into saturation at higher energies, depending on the difference in $Z$ between the target and the product.

This effect will be illustrated on the example of rhenium produced in a uranium compound target. Rhenium is 17 units below uranium. Recently, new types of targets were proposed for extraction of rhenium beams [56]. To take the energy loss and the attenuation of the beam into account in a simplified way, one can divide the target in three equal parts of $80 \mathrm{~g} / \mathrm{cm}^{2}$ each, and calculate the production rate in each segment. Using production cross sections calculated at the energy of the beam in the middle of each segment, and taking into account the reaction rate in each segment,

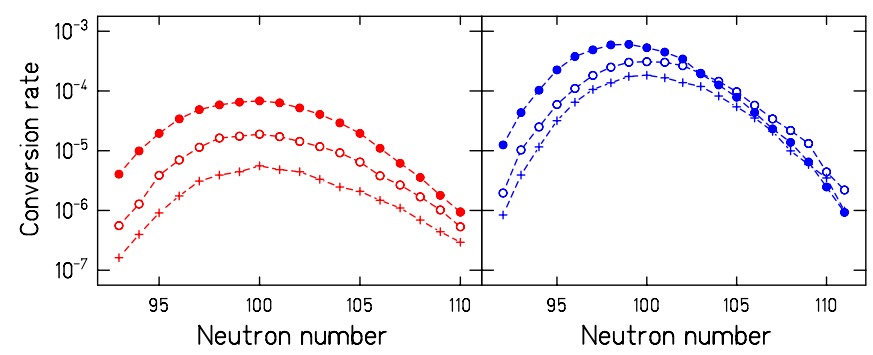

FIG. 26. (Color) Production rates per beam particle for rhenium isotopes in three consecutive uranium-carbide target segments of equal thickness $\left(80 \mathrm{~g} / \mathrm{cm}^{2}\right)$. Left - the case of the proton beam; right - the case of ${ }^{3} \mathrm{He}$ beam. Solid dots - first segment; open dots - second segment; crosses — third segment.

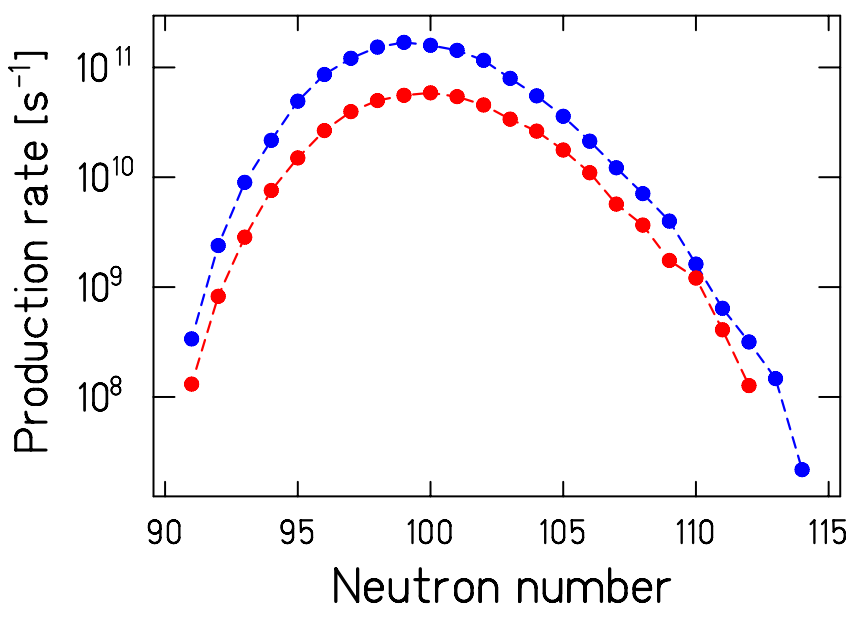

FIG. 27. (Color) In-target production rates of rhenium isotopes $(Z=75)$, in the spallation-evaporation reaction induced by $1 \mathrm{GeV}$ protons (red symbols) and $2 \mathrm{GeV}{ }^{3} \mathrm{He}$ (blue symbols) in a uranium-carbide target. The beam power is $100 \mathrm{~kW}$ in the case of protons, and $50 \mathrm{~kW}$ in the case of ${ }^{3} \mathrm{He}$. A target thickness of $240 \mathrm{~g} / \mathrm{cm}^{2}$ was assumed, beyond which the production drops significantly in both cases.

one can obtain production rates for all the isotopes. The results in the case of the $1 \mathrm{GeV}$ proton and $2 \mathrm{GeV}{ }^{3} \mathrm{He}$ beams are shown in the Fig. 26. The decrease in the production rates along the target is visibly sharper in the case of the proton beam.

Figure 27 compares the maximum obtainable in-target production rates for the isotopic chains of this element with a $1 \mathrm{GeV}$ proton beam or a $2 \mathrm{GeV}{ }^{3} \mathrm{He}$ beam. The beam power has been normalized to $100 \mathrm{~kW}$ for protons and $50 \mathrm{~kW}$ for ${ }^{3} \mathrm{He}$.

Obviously, there is a clear advantage for the production of rhenium in favor of the $2-\mathrm{GeV}$ option by up to a factor of 4 , if the same thermal power density in the production target is considered. The gain factor decreases slightly towards the neutron-rich wing of the isotopic distribution.

The final judgement on the benefit of a $2 \mathrm{GeV}^{3} \mathrm{He}$ beam depends on the size of the gaps in the choice of suitable target material for EURISOL. If suitable targets can be provided by future research and development all over the element range with a maximum distance of 10 units, there is no gain by the $2 \mathrm{GeV}$ option with respect to the $1 \mathrm{GeV}$ option. If one expects that gaps of about 20 units remain, there is a clear advantage up to a factor of 4 for the $2-\mathrm{GeV}$ primary beam for certain elements due to the extended mass range produced.

\section{Production of neutron-deficient isotopes}

To investigate the population of the most neutrondeficient isotopes as the second criterion for the benefit of a $2 \mathrm{GeV}$ primary beam, calculations on a hypothetical production of isotopes of the element number 60 in targets with element numbers 62,72 , and 82 were performed, as 


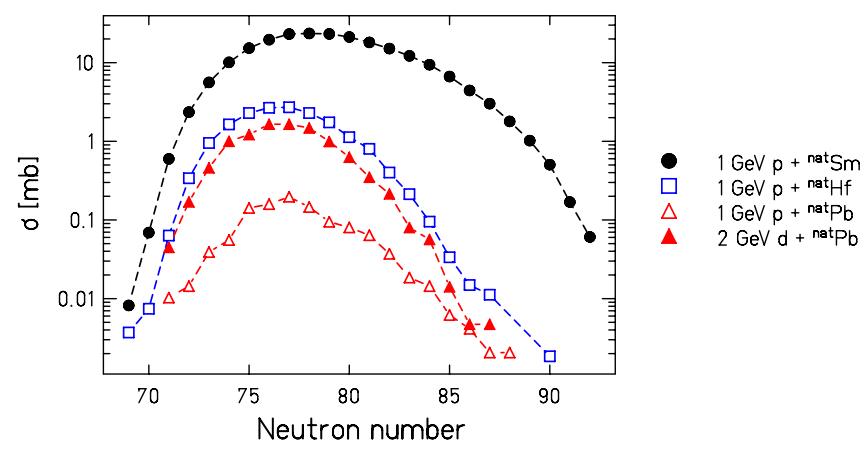

FIG. 28. (Color) Calculated production cross sections of neodymium $(Z=60)$ isotopes by $1 \mathrm{GeV}$ protons and $2 \mathrm{GeV}$ deuterons in different targets.

an example which does not necessarily obey the operating conditions for ISOL targets. Natural isotopic composition of the target materials was assumed. Again, the answer on the benefit of the $2 \mathrm{GeV}$ beam depends on the size of the gap between available target materials. A target element slightly above the desired product gives the highest cross section for all isotopes, including the most neutrondeficient ones. In Fig. 28, this is the case of the target with $Z=62$ for the production of the isotopes of the element number 60 .

If the target element is 22 units above the desired product, the use of the $2 \mathrm{GeV}$ beam has a clear advantage, as already seen in the previous figure. However, a combination of a target element 12 units above the desired fragment with the $1 \mathrm{GeV}$ proton beam gives higher yields, if such a target is available. Thus, if a reasonably close-by target is available, there is no specific gain for the production of neutron-deficient isotopes from increasing the beam energy and using a heavier target.

Our calculations with targets of different isotopic composition have shown that one should prefer to use an even element as a target material if exotic nuclei are to be produced, because it provides a larger number of primordial isotopes. This way, one obtains higher yields on both wings of the isotopic distributions, on the neutron-deficient and on the neutron-rich side.

The results obtained here for the production of neodymium isotopes in targets with $Z$ by 2,12 , and 22 units larger can be generalized to other cases. The essential parameter is the difference in atomic number of the target material and the fragment to be produced.

\section{B. Enhancing IMF emission to produce light neutron- rich nuclides by a $2 \mathrm{GeV}$ primary beam in a fissile target}

The production of intermediate-mass fragments $(Z<$ 30 ) in a fissile target is sensitive to the energy of the incoming beam. To investigate to what extent their production cross sections can be enhanced, we have performed calculations on nuclide production in a ${ }^{238} \mathrm{U}$

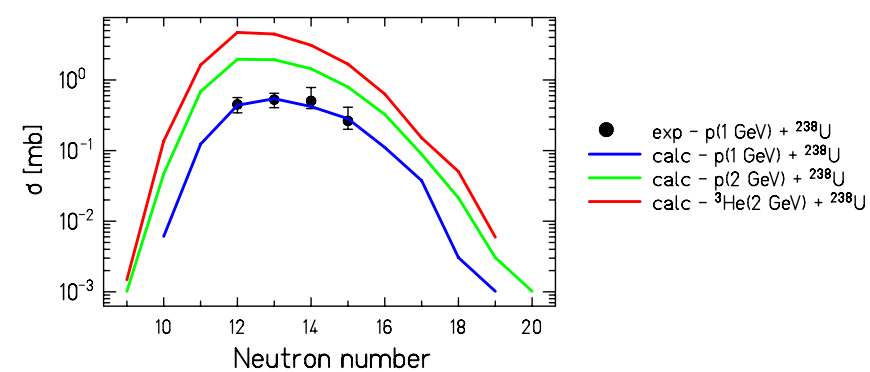

FIG. 29. (Color) Production cross sections of Na in a ${ }^{238} \mathrm{U}$ target interacting with different beams; $1-\mathrm{GeV}$ protons (blue line), 2$\mathrm{GeV}$ protons (green line), and $2-\mathrm{GeV}{ }^{3} \mathrm{He}$ (red line). Calculations were made with INCL4 + ABLA. For comparison, also sodium production cross sections (points) measured at GSI in the reaction ${ }^{1} \mathrm{H}(1 \mathrm{GeV})+{ }^{238} \mathrm{U}[23]$ are shown.

target using $1-\mathrm{GeV}$ proton, $2-\mathrm{GeV}$ proton, and $2-\mathrm{GeV}$ ${ }^{3} \mathrm{He}^{2+}$ beams. The calculations were performed using the intranuclear-cascade code INCL4 [57] coupled to ABLA.

In Fig. 29 we compare production cross sections of sodium calculated using the above-mentioned options. In the same figure, we also show the sodium cross sections from the reaction ${ }^{1} \mathrm{H}(1 \mathrm{GeV})+{ }^{238} \mathrm{U}$ measured at GSI [23]. According to these calculations, with $2-\mathrm{GeV}$ protons we can expect an increase in the production cross sections of IMFs by a factor of $\sim 4$ as compared to the $1-\mathrm{GeV}$ proton option. Moreover, if one would use ${ }^{3} \mathrm{He}$ beam one could expect $\sim 8$ times stronger production of IMF as compared to the standard $1-\mathrm{GeV}$ option. The reason for the additional increase of the IMF production cross sections with the use of ${ }^{3} \mathrm{He}$ projectiles is related to the stronger population of the high-energy tail of the calculated prefragment excitation-energy distribution due to the larger size of the projectile. However, there are no analyzed experimental data yet to confirm this effect.

We can conclude that an increase in the primary-beam energy and, additionally, the use of a ${ }^{3} \mathrm{He}^{2+}$ beam could lead to an important enhancement of light, $Z<30$, neutron-rich nuclides. If normalized to the same deposited thermal energy, this enhancement amounts to a factor of about 2 .

\section{Extended fission-fragment distribution in neutron- induced fission using deuteron-converter neutron source, with $E_{n}=20$ to $80 \mathrm{MeV}$}

The converter-target option using deuterons below $100 \mathrm{MeV}$ differs essentially in one aspect from the $1 \mathrm{GeV}$ proton indirect-target option: The deuteron option provides neutrons with an energy spectrum peaking at about $40 \%$ of the primary-deuteron energy, while the proton option is dominated by evaporated neutrons in the few$\mathrm{MeV}$ range. The different neutron-energy distributions lead to different nuclide distributions. In the deuteron case the gap close to symmetric fission around $Z=46$ is filled up, and the elements in both tails below $Z=30$ and 


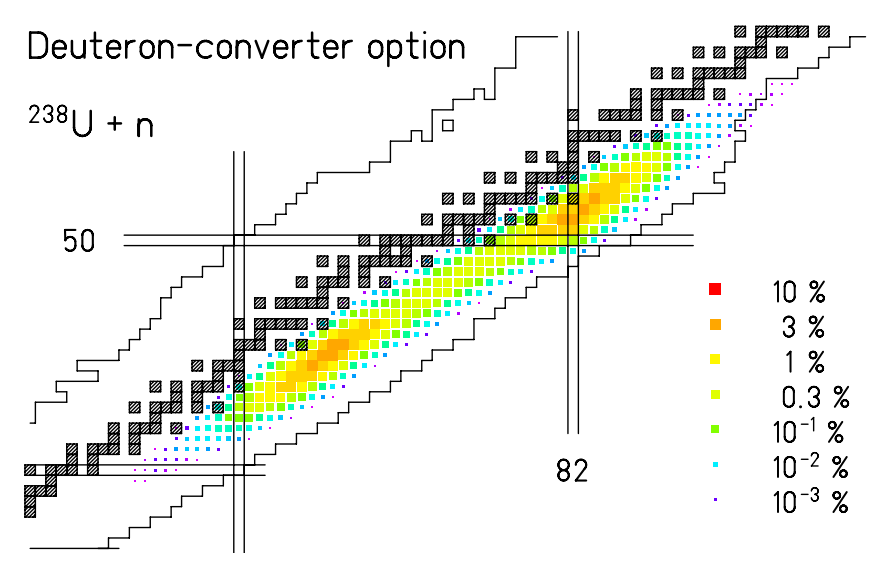

FIG. 30. (Color) In-target nuclide-production yields for a 50$\mathrm{MeV}$ deuteron-converter option, calculated with the ABRABLA code, using the experimental neutron-energy spectrum of Fig. 16 within $10^{\circ}$ emission angle. The yields are given on a color scale in percent. The in-target production rates are obtained by multiplying these yields with the total fission rate.

above $Z=62$ are populated more strongly. All these aspects were already qualitatively discussed in Sec. IVC. The small component of ${ }^{235} \mathrm{U}$ in the natural or depleted uranium does not affect the fission rate or the fission fragment distribution in this case, as there are few thermal neutrons in the spectrum.

In Fig. 30, we present the results of model calculations for the nuclide yields in the deuteron-converter option with a $50 \mathrm{MeV}^{2} \mathrm{H}$ primary beam and a target geometry similar to the one used in SPIRAL 2, which mostly exploits the neutrons produced close to the projectile velocity in forward direction.

The EURISOL high-power target is presently designed to support a primary beam of $1 \mathrm{GeV}$ protons with a beam current of 3 to $4 \mathrm{~mA}$, corresponding to a beam power of 3 to 4 MW [58]. Fission rates induced by secondary neutrons of about $10^{15} \mathrm{~s}^{-1}$ are expected.

In-target nuclide-production rates estimated with the ABRABLA code for the EURISOL high-power target are shown in Fig. 31.

For orientation, we mention two other cases, where deuterons are considered as primary beam.

SPIRAL 2 is designed to provide a fission rate of $10^{13} \mathrm{~s}^{-1}$ with a standard low-density $\mathrm{UC}_{x}$ target with a $40 \mathrm{MeV}$ deuteron beam of $5 \mathrm{~mA}$ or $3 \times 10^{16}$ projectiles per second [59]. This corresponds to a beam power of $200 \mathrm{~kW}$. The fission rate could be increased to $10^{14} \mathrm{~s}^{-1}$ by using a high-density $\mathrm{UC}_{x}$ target.

In the latest design of RIA, a deuteron beam up to $800 \mathrm{MeV}$ and a beam power of $100 \mathrm{~kW}$ were considered as one of the different options, however due to the target geometry used, the production mostly relies on fission by low-energy neutrons in the range from 0.5 to $3 \mathrm{MeV}$ [60], thus providing a nuclide distribution similar to the one obtained in the EURISOL proton-converter option.

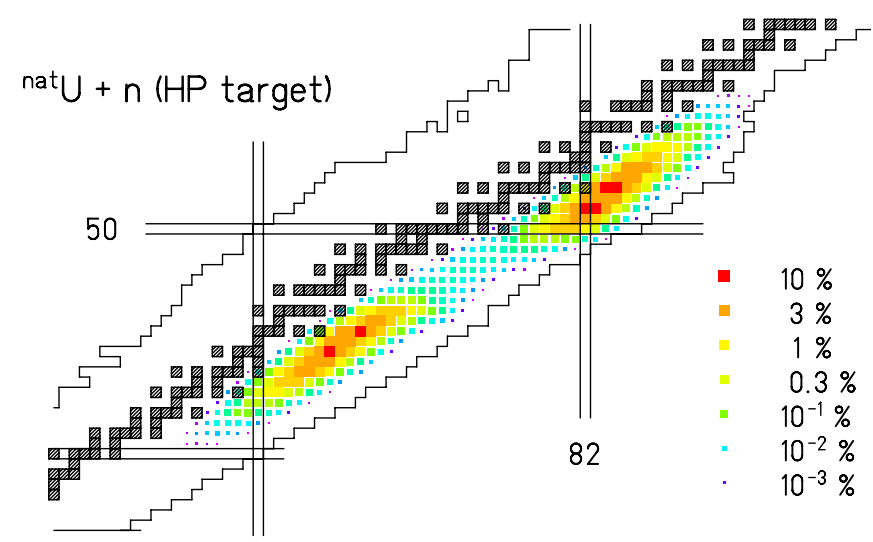

FIG. 31. (Color) In-target nuclide-production yields for the EURISOL 2-step high-power option calculated with the ABRABLA code, using the neutron-energy spectrum of Fig. 14. The yields are given on a color scale in percent. The in-target production rates are obtained by multiplying these yields with the total fission rate.

\section{Exploiting general properties of fragmentation induced by heavy-ions}

As discussed in Sec. IV D, it is not clear to which extent the broader isotopic distributions in fragmentation reactions induced by heavy-ions are caused by nuclear-reaction aspects, as supposed by Ravn et al. [9], and to which extent by lower decay losses.

A detailed study of the decay-loss aspect of the increase in the exotic-nuclide yields is beyond the scope of this work. Such a detailed study should carefully consider the different limitations, e.g., due to available primary-beam intensities and the affordable heat load in the target.

\section{E. Converter-catcher-ISOL scenario using heavy-ion beams}

Estimates of attainable secondary-beam intensities by fragmentation of heavy ions were performed by the targetand-ion-source group in the EURISOL RTD report (Appendix C, Sec. 2.2.3) [4]. The calculations were performed assuming a multibeam driver delivering any heavyion beam with $A / q=6$ or 3 at 166.5 or $333 A \mathrm{MeV}$, respectively, impinging on a carbon converter target in which most of the primary beam energy is lost. The reaction products are stopped in a diffusion catcher, which is placed just behind the converter and can in principle consist of any material, providing best conditions for extraction. The production yields were only calculated in the target/converter, because only projectile fragmentation was taken into account. The projectile fragment yields were estimated via EPAX. Losses by secondary reactions of the radioactive fragments during their stopping and transfer were taken into account. The best suited projectile was chosen, to give the highest yields and for which ECRIS technology may be able to deliver the beam intensities assumed for the calculation. Results of these calculations 


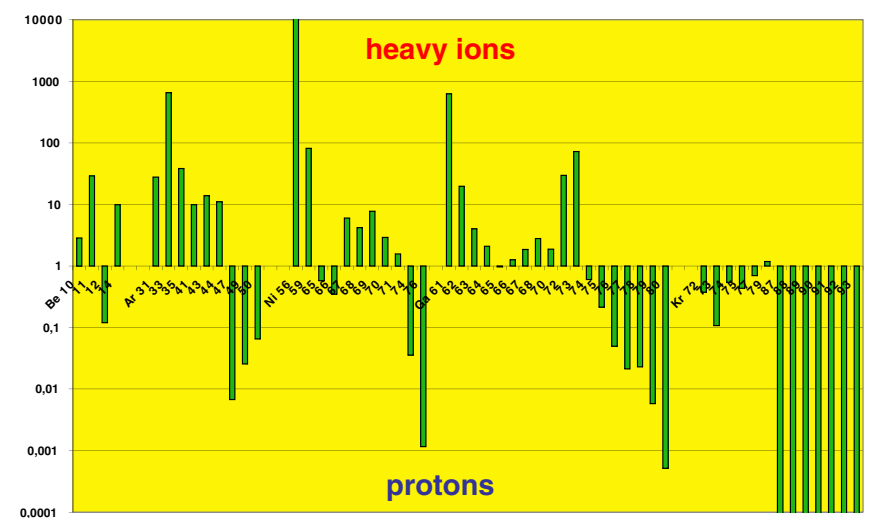

FIG. 32. (Color) Comparison of the yields obtained with a beam of $1 \mathrm{GeV}$ protons $(100 \mu \mathrm{A})$ and with an optimum heavy-ion beam. See text for details. The figure is taken from Ref. [61].

showed that for light nuclei heavy ions present an advantage of 1 order of magnitude or more in the in-target yields (essentially due to the use of the converter-catcher method for light heavy ions) as compared to the proton option. For heavier ions, this advantage disappears and the tendency is reversed. Thus, a multibeam driver, with both protons and light heavy ions could extend the converter-catcher concept over the whole chart of the nuclides.

The gain factors obtained in this elaborate study for the in-target production, compared to a $100 \mu \mathrm{A}$ beam of $1 \mathrm{GeV}$ protons, are shown in Fig. 32. These gain factors should be adapted to the primary-beam intensities and energies obtainable with the different scenarios for the driver accelerator listed in Sec. II2. Disregarding changes in the nuclear-reaction characteristics that may be important below $100 \mathrm{~A} \mathrm{MeV}$ and in primary-beam intensities, the nuclide production rates scale with the usable target thickness, which strongly reduces with decreasing beam energy. Table I lists the energies and the relative total nuclearreaction rates in the usable target thickness for a few beamenergy values. The production rates for specific nuclides roughly scale with this quantity. That is, the in-target production yields with a ${ }^{40} \mathrm{Ar}$ beam decrease by a factor of about $0.5 / 40=1 / 80$, when the energy is reduced from $333 \mathrm{~A} \mathrm{MeV}$, as assumed for the estimations in Fig. 32, to $28 \mathrm{~A} \mathrm{MeV}$, if the beam intensity remains the same.

Finally, it should be stressed that the investigations of the target-and-ion-source group reported in the EURISOL RTD report (Appendix C, Sec. 2.2.3) [4] assumed that the overall ISOL efficiencies obtainable for the heavy-ion fragmentation option are equal to the ones of the $1-\mathrm{GeV}$ proton direct-target option of EURISOL. For a general judgment on this assumption, appropriate targets, which are now available at SPIRAL mostly for noble gases, need to be developed for the heavy-ion fragmentation option.

As a specific case, we have also considered more closely the production of ${ }^{18} \mathrm{Ne}$ in three different scenarios as presented in Table II. The option using a ${ }^{20} \mathrm{Ne}$ beam is compared to two scenarios using a $1 \mathrm{GeV}$ proton beam with $\mathrm{MgO}$ and Ta targets. The initial energy of $500 \mathrm{~A} \mathrm{MeV}$ and $A / q=2$, as envisioned in one of the options for the EURISOL driver accelerator, was considered. In the case of light targets, the production cross sections are estimated

TABLE I. Fraction $R$ of the projectiles, which react in a carbon target with a thickness of $80 \%$ of the range of the corresponding projectile, for different beam energies. The beam energies correspond to the different EURISOL driver-accelerator options listed in Sec. II. Cases corresponding to the comparison shown in Fig. 32 are printed in bold.

\begin{tabular}{|c|c|c|c|c|c|c|c|c|c|}
\hline \multirow[b]{2}{*}{ Projectile } & \multirow[b]{2}{*}{$\begin{array}{c}E / A \\
{[\mathrm{MeV}]}\end{array}$} & \multirow[b]{2}{*}{$\begin{array}{c}I \\
{[\mathrm{~mA}]}\end{array}$} & \multicolumn{7}{|c|}{$R[\%]$} \\
\hline & & & $\begin{array}{c}28 A \\
\mathrm{MeV}\end{array}$ & $\begin{array}{c}43 A \\
\mathrm{MeV}\end{array}$ & $\begin{array}{l}100 A \\
\mathrm{MeV}\end{array}$ & $\begin{array}{l}150 A \\
\mathrm{MeV}\end{array}$ & $\begin{array}{c}166.5 A \\
\mathrm{MeV}\end{array}$ & $\begin{array}{l}333 A \\
\mathrm{MeV}\end{array}$ & $\begin{array}{l}500 A \\
\mathrm{MeV}\end{array}$ \\
\hline $\mathrm{B}, \mathrm{C}, \mathrm{O}$ & 333 & 1 & $\sim 1$ & $\sim 2$ & $8-13$ & $16-24$ & $20-28$ & $50-65$ & $73-86$ \\
\hline $\mathrm{Ar}$ & 333 & 0.5 & $\sim 0.5$ & $\sim 1$ & $\sim 7$ & $\sim 13$ & $\sim 15$ & $\sim \mathbf{4 0}$ & $\sim 63$ \\
\hline $\mathrm{Ca}$ & 166.5 & 0.5 & $\sim 0.5$ & $\sim 1$ & $\sim 5$ & $\sim 11$ & $\sim 13$ & $\sim 35$ & $\sim 55$ \\
\hline $\mathrm{Ni}$ & 166.5 & 0.4 & $\sim 0.5$ & $\sim 1$ & $\sim 5$ & $\sim 10$ & $\sim 12$ & $\sim 32$ & $\sim 53$ \\
\hline $\mathrm{Zn}, \mathrm{Ge}, \mathrm{Se}, \mathrm{Kr}, \mathrm{Ag}$ & 166.5 & 0.3 & $\sim 0.5$ & $\sim 1$ & $\sim 5$ & $9-10$ & $\sim 11$ & $\sim 30$ & $\sim 50$ \\
\hline $\mathrm{Sn}, \mathrm{Te}, \mathrm{Xe}, \mathrm{Ce}$ & 166.5 & 0.2 & $\sim 0.5$ & $\sim 1$ & $\sim 4$ & $\sim 8$ & $\sim \mathbf{1 0}$ & $\sim 27$ & $\sim 45$ \\
\hline
\end{tabular}

TABLE II. Comparison of several scenarios to produce a ${ }^{18} \mathrm{Ne}$ secondary beam. See text for details.

\begin{tabular}{|c|c|c|c|c|c|}
\hline Reaction & $\sigma\left({ }^{18} \mathrm{Ne}\right)[\mathrm{mb}]$ & $\begin{array}{c}\text { Reaction rate in } \\
\text { target }[\%]\end{array}$ & $\begin{array}{c}\text { In-target } \\
\text { yield }\left[\mathrm{s}^{-1}\right] \\
(I=100 \mu \mathrm{A})\end{array}$ & $\begin{array}{c}\text { Overall } \\
\text { efficiency [\%] }\end{array}$ & $\begin{array}{c}\text { RIB intensity } \\
{\left[\mathrm{s}^{-1}\right]}\end{array}$ \\
\hline${ }^{20} \mathrm{Ne}+\mathrm{C}$ & 2.3 & 73 & $3.4 \times 10^{10}$ & 3 & $1 \times 10^{9}$ \\
\hline$p+\mathrm{MgO}$ & 0.7 & 97 & $1.4 \times 10^{11}$ & 3 & $4.2 \times 10^{9}$ \\
\hline$p+\mathrm{Ta}$ & $4.9 \times 10^{-4}$ & 30 & $3.0 \times 10^{7}$ & 13 & $4.0 \times 10^{6}$ \\
\hline
\end{tabular}


using the EPAX parametrization. The validity of EPAX for the reaction channels close to the projectile was verified using data from the reaction ${ }^{56} \mathrm{Fe}+p$, obtained at GSI $[18,19]$. EPAX reproduces the data well for channels with removal of up to six protons independent of the beam energy which ranged from 300 to $1500 \mathrm{~A} \mathrm{MeV}$. For heavier targets (tantalum and uranium carbide), the ABRABLA code was used to calculate the cross sections. The reaction rate in the target was estimated using the code AMADEUS [62], taking into account that, in the case of a heavy target, only a limited target thickness is usable due to the rapidly decreasing cross sections for complex-fragment emission. An initial beam intensity of $100 \mathrm{~kW}$ was considered in all cases. The high deposited power density of the Ne beam might be handled by using the rotating-wheel target design [4] (Appendix C, Sec. 2.2.3). The overall efficiency for the reaction ${ }^{20} \mathrm{Ne}+\mathrm{C}$ was estimated using the experimental value obtained at SPIRAL, as reported in [4] (Appendix C, Sec. 2.2.3), for the reaction $p+\mathrm{MgO}$ the same value was taken, assuming an ECRIS ion source, and for the reaction $p+$ Ta a rough estimate was obtained using the formula of Lukić et al. [16] with global parameters $\varepsilon_{s}=0.3, \alpha=1.5$ and $\tau=2 \mathrm{~s}$. The estimated RIB intensity for the reaction ${ }^{20} \mathrm{Ne}+\mathrm{C}$ thus appears lower by a factor of 4 than for the reaction $p+\mathrm{MgO}$ and more than 2 orders of magnitude higher than in the reaction $p+$ Ta.

In Table III we show the results of a similar quantitative comparison of the production of the nuclide ${ }^{14} \mathrm{Be}$ for the reaction ${ }^{18} \mathrm{O}+\mathrm{C}$ and several proton-beam options that are currently used at ISOLDE (Ta, $\mathrm{UC}_{x}$ ), or could be used in the future $\left(\mathrm{SiC}_{x}\right)$. The production cross sections and the reaction rates in the target were estimated in the same way as in the previous example. As no data on the overall efficiency of beryllium are available, only in-target yields are considered. The energy of the ${ }^{18} \mathrm{O}$ beam was again fixed to $500 A \mathrm{MeV}$, even if the $A / q$ ratio in this case slightly exceeds two. The RIB intensity in the heavy-ion option is 2 orders of magnitude higher than with the silicon-carbide or tantalum targets, but of the same order of magnitude as with the uranium-carbide target.

Thus, in both cases, the heavy-ion option with $500 \mathrm{~A} \mathrm{MeV}$ seems to lead to a final RIB intensity of the same order of magnitude as in the baseline scenario, with a

TABLE III. Comparison of several scenarios to produce a ${ }^{14} \mathrm{Be}$ secondary beam. See text for details.

\begin{tabular}{lccc}
\hline \hline Reaction & $\sigma\left({ }^{14} \mathrm{Be}\right)[\mathrm{mb}]$ & $\begin{array}{c}\text { Reaction rate in } \\
\text { target }[\%]\end{array}$ & $\begin{array}{c}\text { In-target } \\
\text { yield }\left[\mathrm{s}^{-1}\right] \\
(I=100 \mu \mathrm{A})\end{array}$ \\
\hline${ }^{18} \mathrm{O}+\mathrm{C}$ & $2 \times 10^{-3}$ & 85 & $3.4 \times 10^{7}$ \\
$p+\mathrm{SiCx}$ & $2.6 \times 10^{-6}$ & 97 & $4.8 \times 10^{5}$ \\
$p+\mathrm{Ta}$ & $3.3 \times 10^{-6}$ & 30 & $2.2 \times 10^{5}$ \\
$p+\mathrm{UCx}$ & $1.7 \times 10^{-4}$ & 30 & $1.2 \times 10^{7}$ \\
\hline \hline
\end{tabular}

carefully chosen target, and does not offer any significant advantage over it.

\section{F. Production of neutron-rich nuclides in heavy-ion reactions in the Fermi-energy regime related to nucleon exchange between target and projectile \\ 1. Production cross sections of nuclei around ${ }^{78} \mathrm{Ni}$.}

Figure 33 shows a comparison of the production of element $\mathrm{Zn}$ and below. The experimental spallation cross sections for the reaction of $1 \mathrm{GeV}$ protons with ${ }^{238} \mathrm{U}$ [22] is represented by symbols and solid lines, representing Gaussian fits of the experimental mass distributions. The experimental cross sections for the reaction of $1 \mathrm{GeV}$ protons with ${ }^{238} U$ [22] were also compared to values calculated using the ABRABLA code. It appears that the calculation for elements below $\mathrm{Zn}$ (in the light fission tail) overestimates the production cross sections for neutron-rich nuclei, and thus a representation by Gaussian fits of experimental mass distributions was chosen.

The estimates of production cross sections in fragmentation of stable nuclei, obtained using EPAX by optimization for each nucleus separately are represented by blue lines. The production cross sections for the reactions of ${ }^{86} \mathrm{Kr},{ }^{82} \mathrm{Se}+{ }^{64} \mathrm{Ni}$ at $25 \mathrm{~A} \mathrm{MeV}$, were calculated using the modified version [55] of the deep-inelastic transfer (DIT) code of Tassan-Got [52] (red and green lines). One can see that the estimated optimum fragmentation cross sections essentially follow the experimental trend as described by the Gaussian fits. On the other hand, the production cross sections in the reactions of ${ }^{86} \mathrm{Kr},{ }^{82} \mathrm{Se}+{ }^{64} \mathrm{Ni}$ at $25 \mathrm{~A} \mathrm{MeV}$ appear to dramatically overshoot the experimental trend for spallation of ${ }^{238} \mathrm{U}$. In this aspect it is worthwhile to mention that the calculation for the reactions of ${ }^{86} \mathrm{Kr}+$ ${ }^{64} \mathrm{Ni}$ at $25 \mathrm{~A} \mathrm{MeV}$, used in Fig. 33, correctly reproduces the experimental cross sections, measured at angles below 3 degrees, as documented in Fig. 34 (the discrepancies at the proton-rich side are caused by restricted $\mathrm{B} \rho$ coverage for such products in the experiment, which was focused primarily on neutron-rich products $[45,55])$.

\section{Estimate of the intensity of secondary beams}

Figure 35 shows estimated RIB intensities of nuclides around ${ }^{78} \mathrm{Ni}$ using the production cross sections presented above. The solid lines represent estimated RIB intensities based on experimental spallation cross sections (Gaussian fits of measured mass distributions) for the reaction of $1 \mathrm{GeV}$ protons with ${ }^{238} \mathrm{U}$ [22]. A primary proton-beam intensity of $100 \mu \mathrm{A}$ impinging directly on the ${ }^{238} \mathrm{U}$ target was assumed. The target thickness corresponding to one interaction length is assumed. The efficiencies are estimated based on the work of Lukić et al. [16], where the RIB intensities for alkali elements, halogenides, and noble gases measured at ISOLDE using thick $\mathrm{UC}_{x}$ target are 

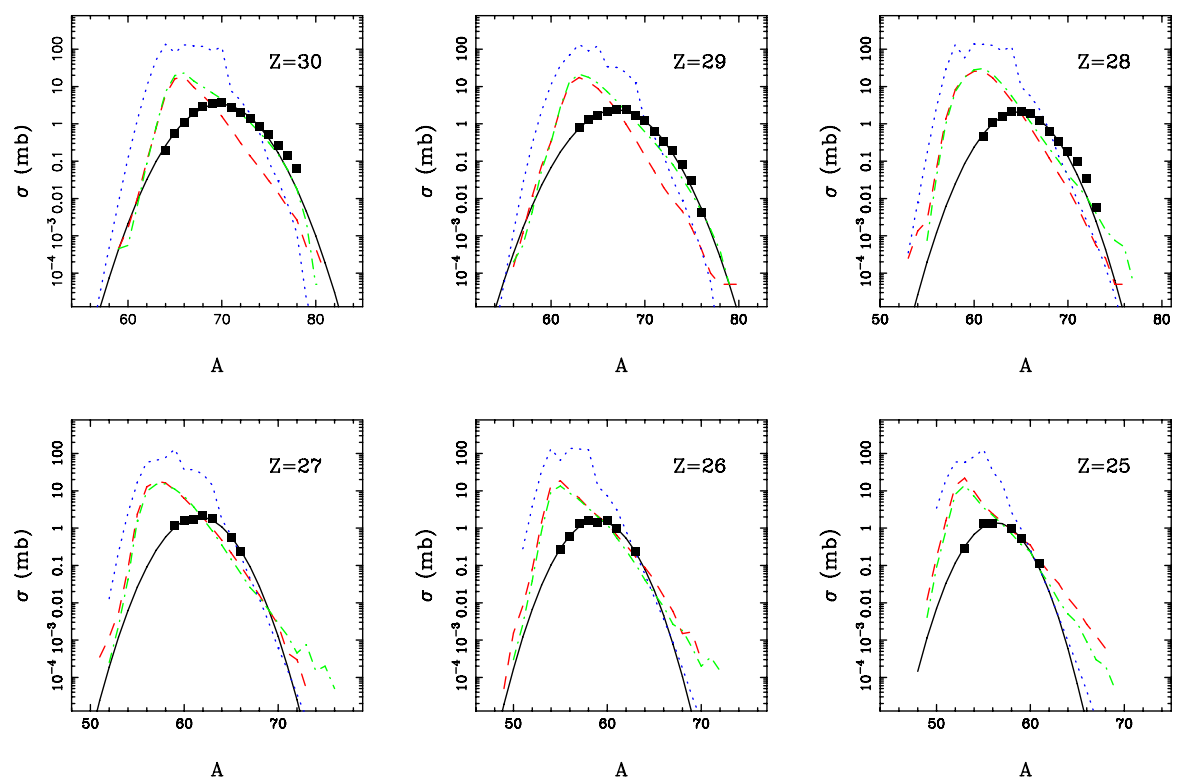

FIG. 33. (Color) Production cross sections of isotopes of $\mathrm{Zn}$ and elements below. Symbols - experimental data for reaction of $1 \mathrm{GeV}$ protons with ${ }^{238} \mathrm{U}$ [22]; solid lines-Gaussian fits of experimental mass distributions; blue lines - estimates of production cross sections in fragmentation of stable nuclei, obtained using EPAX by optimization for each nucleus separately; red and green lines production cross sections for reactions of ${ }^{86} \mathrm{Kr},{ }^{82} \mathrm{Se}+{ }^{64} \mathrm{Ni}$ at $25 \mathrm{~A} \mathrm{MeV}$, calculated using the modified version [55] of the deepinelastic transfer code.

compared to in-target yields calculated for a uranium target using ABRABLA. We adopt the parameters $\varepsilon_{s}=0.3, \alpha=1$, and $\tau=10 \mathrm{~s}$ [see Eq. (1)], which represent a global upper limit on extraction efficiencies for various chemical groups treated in work [16].

The blue dotted line represents estimated RIB intensities for the process of fragmentation of stable beams (the beam species optimized for each nuclide). The primary-beam intensity $100 \mathrm{p} \mu \mathrm{A}$ was assumed. As discussed in the EURISOL Report resulting from the feasibility study RTD, Appendix C, Sec. 2.2.3, the intensity of several hundreds of $\mathrm{p} \mu \mathrm{A}$ for beams up to krypton can be considered as achievable for the foreseeable future. The beam energy $500 \mathrm{~A} \mathrm{MeV}$ was considered, high enough to allow
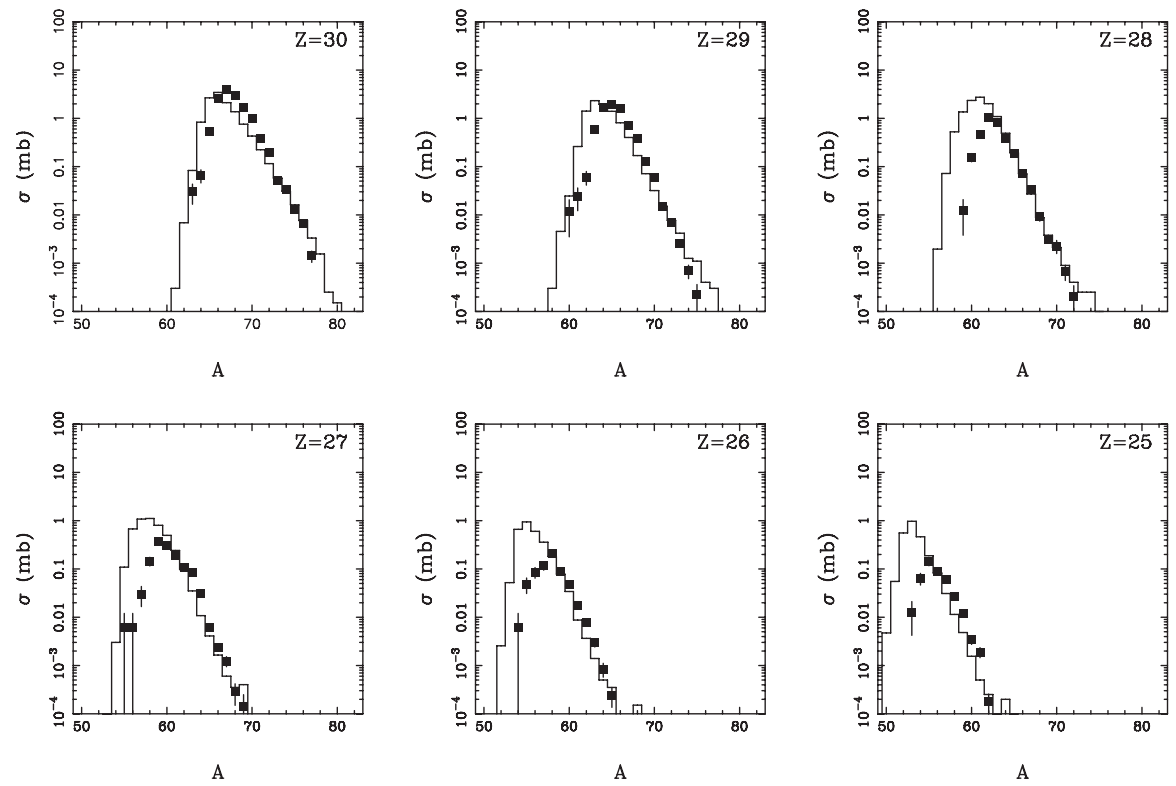

FIG. 34. Experimental production cross sections of isotopes of $\mathrm{Zn}$ and elements below measured in the reaction ${ }^{86} \mathrm{Kr}+{ }^{64} \mathrm{Ni}$ at $25 \mathrm{~A} \mathrm{MeV}$ for angles below 3 degrees [45] (symbols), compared to the results of calculation [55] (lines). 

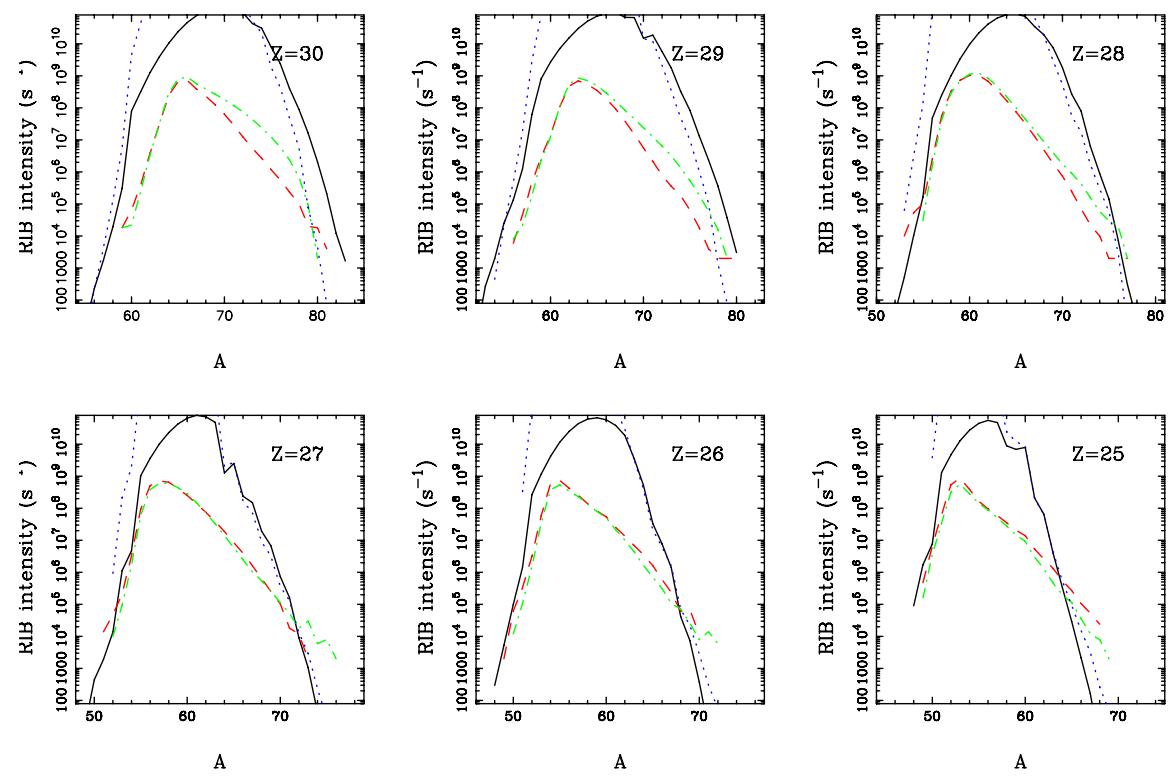

FIG. 35. (Color) Estimated RIB intensities of nuclides around ${ }^{78} \mathrm{Ni}$ for primary beam intensity $100 \mathrm{p} \mu \mathrm{A}$. Solid lines - reaction of $1 \mathrm{GeV}$ proton beam with ${ }^{238} \mathrm{U}$ target; blue lines — fragmentation of stable nuclei; red and green lines - reactions of ${ }^{86} \mathrm{Kr}$, ${ }^{82} \mathrm{Se}+{ }^{64} \mathrm{Ni}$ at $25 \mathrm{~A} \mathrm{MeV}$.

the use of a thick target leading to reaction rates close to the proton-beam case. In particular, for the ${ }^{86} \mathrm{Kr}$ beam with the energy of $500 \mathrm{~A} \mathrm{MeV}$ the reaction rate in the target will approach $60 \%$, on the other hand the beam power will exceed $4 \mathrm{MW}$ which is probably at the limit of what the converter may sustain. The beam energy $500 \mathrm{~A} \mathrm{MeV}$ was not considered in the EURISOL driver heavy-ion options for beams up to krypton, where for $A / q=3$ nuclei the maximum considered energy is $100 \mathrm{~A} \mathrm{MeV}$, which however would lead to a loss of the in-target yield by 1 order of magnitude. The extraction efficiencies were determined as in the previous case.

Red and green lines represent estimated RIB intensities for reactions of ${ }^{86} \mathrm{Kr},{ }^{82} \mathrm{Se}+{ }^{64} \mathrm{Ni}$ at $25 \mathrm{~A} \mathrm{MeV}$. As in the work [45], a rather moderate target thickness of $20 \mathrm{mg} / \mathrm{cm}^{2}$ was assumed. The primary-beam intensity of $100 \mathrm{p} \mu \mathrm{A}$ was chosen, and a transport efficiency of $30 \%$ was assumed for the large-acceptance separator, thus representing the in-flight option. The angular coverage of the large-acceptance separator up to 10 degrees would be required in order to cover the most populated part of the angular distribution. The results for the $1 \mathrm{GeV}$ proton beam and heavy-ion beam fragmentation are similar and dominate at the neutron-rich side of elements above Ni. However, for the extremely neutron-rich nuclides of nickel and below, the reactions with a relatively thin target and beam energy of $25 \mathrm{~A} \mathrm{MeV}$ appear to lead to higher RIB intensities than in other scenarios, due to the high in-target yields. Nevertheless, the quality of such secondary beams would be lower than in other cases. The use of a gas cell may be an alternative, leading to better beam quality, provided that losses will not increase dramatically, which is an open question needing further research and development.

\section{CONCLUSION}

A number of approaches for the production of radioactive beams have been examined with regard to their potential to be used as possible additional options for the EURISOL project in order to enhance the production of specific nuclear species with respect to the baseline $1-\mathrm{GeV}$ proton case. We found that the spallation of suitable target material by $1 \mathrm{GeV}$ protons and the fission induced by secondary neutrons in a uranium target provide overall high intensities for secondary beams almost all over the chart of the nuclides.

Still, there are cases where the $1 \mathrm{GeV}$ proton beam together with the restrictions of the ISOL method in view of suitable target material do not allow optimizing the reaction parameters. In addition, low overall efficiencies for certain elements let gaps in available beams. Some of these problems can be overcome by providing heavier projectiles and/or higher beam energies: (i) A $2 \mathrm{GeV}$ ${ }^{3} \mathrm{He}^{2+}$ beam would fill the gaps in the nuclide production given by the limited choice of ISOL target materials. This would lead to gain factors up to a factor of 4 , in particular, for the production of neutron-deficient isotopes of many elements. (ii) A $2 \mathrm{GeV}^{3} \mathrm{He}^{2+}$ beam would increase the production of neutron-rich isotopes of light to mediumheavy elements $(Z<30)$ by about a factor of 2 . The deuteron-converter option with a primary-beam energy 
above $40 \mathrm{MeV}$ provides fission-fragment nuclide distributions with appreciably higher fission yields (normalized to the total number of fission events in the target) for elements between technetium $(Z=43)$ and indium $(Z=49)$, below germanium $(Z=32)$, and above neodymium $(Z=60)$ compared to the standard EURISOL high-power-target option. However, only part of this advantage can be used, since many of the enhanced elements are poorly released from ISOL targets. (iii) Fragmentation of heavy-ion projectiles provides higher in-target yields for some neutrondeficient isotopes of light elements and presumably higher overall ISOL efficiencies for short-lived isotopes. It can also be useful to overcome limitations in the choice of the target material in the standard proton option and to divide production target and catcher. The gain factors depend strongly on the beam energy. The deposited power density might be a limiting factor in this scenario. (iv) Because of nucleon exchange between projectile and target, heavy-ion reactions in the Fermi-energy regime (around 20 to $30 \mathrm{~A} \mathrm{MeV}$ ) provide a substantial benefit for the production of neutron-rich isotopes of elements outside the main fission region. In order to take advantage of this production mechanism, several experimental problems, i.e., large angular distributions of fragments must be overcome.

The listed approaches require extended capabilities of the driver accelerator. Thus, the benefit of the options discussed in this report should be carefully considered in view of the extra cost involved.

The present work should be considered as preliminary. First, not all options have been investigated in full quantitative detail. Further on, we could not consider in a conclusive way the possible advance of technological limits, on, e.g., currents provided by the ion source or heat load in the target, until the time EURISOL is constructed. Nevertheless, we hope that this report gives first guidance to the problem and stimulates a fruitful discussion on the questions that remain open.

\section{ACKNOWLEDGMENTS}

We acknowledge the financial support of the European Community under the FP6 "Research Infrastructure Action-Structuring the European Research Area," EURISOL DS Project, Contract No. 515768 RIDS. The EC is not liable for any use that may be made of the information contained herein. We would like to thank Ulli Köster for bringing to our attention many of the ideas developed in the ISOLDE community, as well as for many stimulating remarks and careful reading of the manuscript. We also acknowledge valuable discussions with Wolfgang Mittig on the converter-catcher-ISOL scenario using heavy-ion beams.

[1] http://www.eurisol.org
[2] http://isolde.cern.ch

[3] H. L. Ravn, P. Bricault, G. Ciavola, P. Drumm, B. Fogelberg, E. Hagebo, M. Huyse, R. Kirchner, W. Mittig, A. Mueller, H. Nifenecker, and E. Roeckl, Nucl. Instrum. Methods Phys. Res., Sect. B 88, 441 (1994).

[4] http://www.ganil.fr/eurisol/Final_Report.html

[5] http://www.orau.org/ria

[6] J. Nolen, in Proceedings of the Third International Conference on Radioactive Nuclear Beams, East Lansing, 1993, edited by D. J. Morissey.

[7] E. Cottereau, Nucl. Phys. A701, 78 (2002).

[8] U. Köster, Radiochim. Acta 89, 749 (2001).

[9] H.L. Ravn, Nucl. Instrum. Methods Phys. Res., Sect. B 26, 72 (1987).

[10] K. Helariutta, J. Benlliure, M. V. Ricciardi, and K.-H. Schmidt, Eur. Phys. J. A 17, 181 (2003).

[11] http://www-aix.gsi.de/\%7Emsep/ionsource.html

[12] D.C. Stracener, http://www.phy.ornl.gov/hribf/ usersgroup/news/spring2_01.html

[13] R. Kirchner et al., Nucl. Instrum. Methods Phys. Res., Sect. A 234, 224 (1985).

[14] R. Kirchner et al., Nucl. Instrum. Methods Phys. Res., Sect. A 247, 265 (1986).

[15] R. Kirchner et al., Nucl. Instrum. Methods Phys. Res., Sect. B 26, 204 (1987).

[16] S. Lukic, F. Gevaert, A. Kelic, M.V. Ricciardi, K.-H. Schmidt, and O. Yordanov, Nucl. Instrum. Methods Phys. Res., Sect. A 565, 784 (2006).

[17] C. L. Jiang, B. B. Back, I. Gomes, A. M. Heinz, J. Nolen, K.E. Rehm, G. Savard, and J.P. Schiffer, Nucl. Instrum. Methods Phys. Res., Sect. A 492, 57 (2002).

[18] C. Villagrasa-Canton, Ph.D. thesis, Université de Paris XI, 2003.

[19] P. Napolitani, K.-H. Schmidt, A. S. Botvina, F. Rejmund, L. Tassan-Got, and C. Villagrasa, Phys. Rev. C 70, 054607 (2004).

[20] P. Napolitani, Ph.D. thesis, Université Paris XI, 2004.

[21] J. Taieb, K.-H. Schmidt, L. Tassan-Got, P. Armbruster, J. Benlliure, M. Bernas, A. Boudard, E. Casarejos, S. Czajkowski, T. Enqvist, R. Legrain, S. Leray, B. Mustapha, M. Pravikoff, F. Rejmund, C. Stephan, C. Volant, and W. Wlazlo, Nucl. Phys. A724, 413 (2003).

[22] M. Bernas, P. Armbruster, J. Benlliure, A. Boudard, E. Casarejos, S. Czajkowski, T. Enqvist, R. Legrain, S. Leray, B. Mustapha, P. Napolitani, J. Pereira, F. Rejmund, M. V. Ricciardi, K.-H. Schmidt, C. Stéphan, J. Taieb, L. TassanGot, and C. Volant, Nucl. Phys. A725, 213 (2003).

[23] M. V. Ricciardi, P. Armbruster, J. Benlliure, M. Bernas, A. Boudard, S. Czajkowski, T. Enqvist, A. Kelic, S. Leray, R. Legrain, B. Mustapha, J. Pereira, F. Rejmund, K.-H. Schmidt, C. Stephan, L. Tassan-Got, C. Volant, and O. Yordanov, Phys. Rev. C 73, 014607 (2006).

[24] P. Armbruster, J. Benlliure, M. Bernas, A. Boudard, E. Casarejos, S. Czajkowski, T. Enqvist, S. Leray, P. Napolitani, J. Pereira, F. Rejmund, M.-V. Ricciardi, K.-H. Schmidt, C. Stephan, J. Taieb, L. Tassan-Got, and C. Volant, Phys. Rev. Lett. 93, 212701 (2004).

[25] J.-J. Gaimard and K.-H. Schmidt, Nucl. Phys. A531, 709 (1991).

[26] A. R. Junghans, M. de Jong, H.-G. Clerc, A. V. Ignatyuk, G. A. Kudyaev, and K.-H. Schmidt, Nucl. Phys. A629, 635 
(1998).

[27] J. Benlliure, A. Grewe, M. de Jong, K.-H. Schmidt, and S. Zhdanov, Nucl. Phys. A628, 458 (1998).

[28] B. Jurado, K.-H. Schmidt, and J. Benlliure, Phys. Lett. B 553, 186 (2003).

[29] J. Benlliure, K.-H. Schmidt, D. Cortina-Gil, T. Enqvist, F. Farget, A. Heinz, A. R. Junghans, J. Pereira, and J. Taieb, Nucl. Phys. A660, 87 (1999).

[30] S. B. Kaufmann and E. P. Steinberg, Phys. Rev. C 22, 167 (1980).

[31] B. Fernandez-Dominguez, P. Armbruster, L. Audouin, J. Benlliure, M. Bernas, A. Boudard, E. Casarejos, S. Czajkowski, J.E. Ducret, T. Enqvist, B. Jurado, R. Legrain, S. Leray, B. Mustapha, J. Pereira, M. Pravikoff, F. Rejmund, M. V. Ricciardi, K.-H. Schmidt, C. Stephan, J. Taieb, L. Tassan-Got, C. Volant, and W. Wlazlo, Nucl. Phys. A747, 227 (2005).

[32] L. Audouin, L. Tassan-Got, P. Armbruster, J. Benlliure, M. Bernas, A. Boudard, E. Casarejos, S. Czajkowski, T. Enqvist, B. Fernandez-Dominguez, B. Jurado, R. Legrain, S. Leray, B. Mustapha, J. Pereira, M. Pravikoff, F. Rejmund, M.-V. Ricciardi, K.-H. Schmidt, C. Stephan, J. Taieb, C. Volant, and W. Wlazlo, Nucl. Phys. A768, 1 (2006).

[33] T. Enqvist, W. Wlazlo, P. Armbruster, J. Benlliure, M. Bernas, A. Boudard, S. Czajkowski, R. Legrain, S. Leray, B. Mustapha, M. Pravikoff, F. Rejmund, K.-H. Schmidt, C. Stephan, J. Taieb, L. Tassan-Got, and C. Volant, Nucl. Phys. A686, 481 (2001).

[34] T. Enqvist, P. Armbruster, J. Benlliure, M. Bernas, A. Boudard, S. Czajkowski, R. Legrain, S. Leray, B. Mustapha, M. Pravikoff, F. Rejmund, K.-H. Schmidt, C. Stéphan, J. Taieb, L. Tassan-Got, F. Vivès, C. Volant, and W. Wlazlo, Nucl. Phys. A703, 435 (2002).

[35] M. V. Ricciardi, Phys. Rev. C 73, 014607 (2006).

[36] J. Lettry, R. Catherall, P. Drumm, P. Van Duppen, A. H. M. Evensen, G. J. Focker, A. Jokinen, O.C. Jonsson, E. Kugler, H. Ravn, and ISOLDE Collaboration, Nucl. Instrum. Methods Phys. Res., Sect. B 126, 130 (1997).

[37] S. Lukić, C. Clausen, T. Haarju, and U. Köster (unpublished).

[38] H.-J. Kluge, Isolde user's guide, CERN, Geneva, 1986.

[39] D. Ridikas (private communication).

[40] S. Ménard, M. Mirea, F. Clapier, N. Pauwels, J. Proust, C. Donzaud, D. Guillemaud-Mueller, I. Lhenry, A. C. Mueller, J.A. Scarpaci, and O. Sorlin, Phys. Rev. ST Accel. Beams 2, 033501 (1999).

[41] H. Pentillä, contribution to EURISOL-Task11 meeting, Caen, 2005.

[42] T. Bjornstadt, H.-A. Gustrafsson, B. Jonson, O.C. Jonsson, V. Lindfors, S. Mattsson, A. M. Poskanzer, H. L. Ravn, and D. Schardt, Z. Phys. A 303, 227 (1981).
[43] T. Bjornstadt, L.C. Carraz, H.-A. Gustafsson, J. Heinemeyer, B. Jonson, O.C. Jonsson, V. Lindfors, S. Mattsson, A.M. Poskanzer, and H.L. Ravn, Nucl. Instrum. Methods 186, 391 (1981).

[44] M. Veselsky, Nucl. Phys. A705, 193 (2002).

[45] G. A. Souliotis, M. Veselsky, G. Chubarian, L. Trache, A. Keksis, E. Martin, A. Ruangma, E. Winchester, and S. J. Yennello, Phys. Lett. B 543, 163 (2002).

[46] G. A. Souliotis, D. V. Shetty, M. Veselsky, G. Chubarian, L. Trache, A. Keksis, E. Martin, and S. J. Yennello, Phys. Rev. C 68, 024605 (2003).

[47] M. Veselsky, G. A. Souliotis, G. Chubarian, L. Trache, A. Keksis, E. Martin, A. Ruangma, E. Winchester, and S. J. Yennello, Nucl. Phys. A724, 431 (2003).

[48] G. A. Souliotis, M. Veselsky, G. Chubarian, L. Trache, A. Keksis, E. Martin, D. V. Shetty, and S. J. Yennello, Phys. Rev. Lett. 91, 022701 (2003).

[49] G. A. Souliotis, M. Veselsky, G. Chubarian, and S.J. Yennello, Nucl. Instrum. Methods Phys. Res., Sect. B 204, 166 (2003).

[50] K. Sümmerer and B. Blank, Phys. Rev. C 61, 034607 (2000).

[51] M. Veselsky, R.W. Ibbotson, R. Laforest, E. Ramakrishnan, D. J. Rowland, A. Ruangma, E. M. Winchester, E. Martin, and S. J. Yennello, Phys. Rev. C 62, 064613 (2000).

[52] L. Tassan-Got, Ph.D. Thesis, Orsay, France, 1988 [IPNOT-89-02, 1989]; L. Tassan-Got and C. Stefan, Nucl. Phys. A524, 121 (1991).

[53] J. P. Bondorf, A. S. Botvina, A. S. Iljinov, I. N. Mishustin, and K. Sneppen, Phys. Rep. 257, 133 (1995).

[54] R. Charity, M. A. McMahan, G. J. Wozniak, R. J. McDonald, L. G. Moretto, D. G. Sarantites, L. G. Sobotka, G. Guarino, A. Pantaleo, L. Fiore, A. Gobbi, and K. D. Hildenbrand, Nucl. Phys. A483, 371 (1988).

[55] M. Veselsky and G. A. Souliotis, Nucl. Phys. A765, 252 (2006).

[56] U. Koester, in the Proceedings of The Seventh International Conference on Radioactive Nuclear Beams, Cortina d'Ampezzo, Italy, 2006.

[57] A. Boudard, J. Cugnon, S. Leray, and C. Volant, Phys. Rev. C 66, 044615 (2002).

[58] EURISOL town meeting, Caen, 2005.

[59] http://www.ganil.fr/research/developments/spiral2/index. html

[60] C. L. Jiang, B. B. Back, I. Gomes, A. M. Heinz, J. Nolen, K.E. Rehm, G. Savard, and J.P. Schiffer, Nucl. Instrum. Methods Phys. Res., Sect. A 492, 57 (2002).

[61] A. Villari, contribution to the EURISOL town meeting Orsay, France, 2003.

[62] http://www-w2k.gsi.de/charms/amadeus.htm 